\title{
From Scattered-light to Millimeter Emission: A Comprehensive View of the Gigayear-old System of HD 202628 and its Eccentric Debris Ring
}

\author{
Virginie Faramaz ${ }^{1}$, John Krist ${ }^{1}$, Karl R. Stapelfeldt ${ }^{1}$ (D), Geoffrey Bryden ${ }^{1}$, Eric E. Mamajek ${ }^{1}$ (D), Luca Matrà ${ }^{2}$ (D), Mark Booth ${ }^{3}$ (D), \\ Kevin Flaherty $^{4}$ (D), Antonio S. Hales ${ }^{5,6}$ (D), A. Meredith Hughes ${ }^{7}$, Amelia Bayo, ${ }^{8,9}$ (D), Simon Casassus ${ }^{10,11}$ (D), Jorge Cuadra ${ }^{9,12}$, \\ Johan Olofsson ${ }^{8,9}$ (D), Kate Y. L. Su ${ }^{13}$ (D), and David J. Wilner ${ }^{2}$ (D) \\ ${ }^{1}$ Jet Propulsion Laboratory, California Institute of Technology, 4800 Oak Grove Drive, Pasadena, CA 91109, USA; virginie.c.faramaz@jpl.nasa.gov \\ ${ }^{2}$ Harvard-Smithsonian Center for Astrophysics, 60 Garden Street, Cambridge, MA 02138, USA \\ ${ }^{3}$ Astrophysikalisches Institut und Universitätssternwarte, Friedrich-Schiller-Universität Jena, Schillergäßchen 2-3, D-07745 Jena, Germany \\ ${ }^{4}$ Department of Astronomy and Department of Physics, Williams College, Williamstown, MA 01267, USA \\ 5 Joint ALMA Observatory, Alonso de Córdova 3107, Vitacura 763-0355, Santiago, Chile \\ ${ }^{6}$ National Radio Astronomy Observatory, 520 Edgemont Road, Charlottesville, Virginia, 22903-2475, USA \\ ${ }^{7}$ Department of Astronomy, Van Vleck Observatory, Wesleyan University, Middletown, CT 06459, USA \\ ${ }^{8}$ Instituto de Física y Astronomía, Facultad de Ciencias, Universidad de Valparaíso, Av. Gran Bretaña 1111, Valparaíso, Chile \\ ${ }^{9}$ Núcleo Milenio Formación Planetaria—NPF, Universidad de Valparaíso, Av. Gran Bretaña 1111, Valparaíso, Chile \\ ${ }^{10}$ Departamento de Astronomia, Universidad de Chile, Casilla 36-D, Santiago, Chile \\ ${ }^{11}$ Millennium Nucleus "Protoplanetary Disks," Santiago, Chile \\ ${ }^{12}$ Instituto de Astrofísica, Facultad de Física, Pontificia Universidad Católica de Chile, 782-0436 Santiago, Chile \\ ${ }^{13}$ Steward Observatory, University of Arizona, 933 N. Cherry Avenue, Tucson, AZ 85721, USA \\ Received 2019 March 22; revised 2019 August 1; accepted 2019 August 12; published 2019 September 27
}

\begin{abstract}
We present here new observations of the eccentric debris ring surrounding the Gyr-old solar-type star HD 202628: at millimeter wavelengths with ALMA, at far-infrared wavelengths with Herschel, and in scattered light with the Hubble Space Telescope (HST). The ring inner edge is found to be consistent between ALMA and HST data. As radiation pressure affects small grains seen in scattered-light, the ring appears broader at optical than at millimeter wavelengths. The best fit to the ring seen with ALMA has inner and outer edges at $143.1 \pm 1.7$ au and $165.5 \pm 1.4$, respectively, and an inclination of $57^{\circ} .4 \pm 0.4$ from face-on. The offset of the ring center of symmetry from the star allows us to quantify its eccentricity to be $e=0.09_{-0.01}^{+0.02}$. This eccentric feature is also detected in low resolution Herschel/PACS observations, under the form of a pericenter-glow. Combining the infrared and millimeter photometry, we retrieve a disk grain size distribution index of $\sim-3.4$, and therefore exclude in situ formation of the inferred belt-shaping perturber, for which we provide new dynamical constraints. Finally, ALMA images show four point-like sources that exceed $100 \mu \mathrm{Jy}$, one of them being just interior to the ring. Although the presence of a background object cannot be excluded, we cannot exclude either that this source is circumplanetary material surrounding the belt-shaper, in which case degeneracies between its mass and orbital parameters could be lifted, allowing us to fully characterize such a distant planet in this mass and age regime for the very first time.
\end{abstract}

Key words: circumstellar matter - planetary systems

\section{Introduction}

Debris disks contain solid bodies in a collisional cascade, ranging from $\mathrm{km}$-sized down to micron-sized dust grains. They are remnants of planetary formation processes (see, e.g., the review by Krivov 2010). As examples, our own solar system hosts the Main Asteroid and the Kuiper belts. Extrasolar debris disks were initially detected by the InfraRed Astronomical Satellite through the infrared excess that the micron-sized dust grains add to their host star's emission (Aumann et al. 1984). Since the top reservoir of $\mathrm{km}$-sized bodies is not expected to be replenished, it is expected that debris disks lose luminosity with time, until they become undetectable with our current instruments, and as confirmed by observations (Wyatt 2008; Sierchio et al. 2014). Consequently, debris disks are rarely detected and even more rarely resolved in systems with ages comparable to that of the solar system. Opportunities to investigate the outcome and diversity of mature planetary systems are therefore scarce. One such opportunity is the system of HD 202628.

HD 202628 (HIP 105184) is a G2V star, located at $23.8 \mathrm{pc}$ (Gaia Collaboration et al. 2016, 2018; Bailer-Jones et al. 2018), and has an estimated age of $1.1 \pm 0.4 \mathrm{Gyr}$ (see Appendix A). The debris disk of HD 202628 was first revealed with Spitzer, which detected a significant $70 \mu \mathrm{m}$ excess of nearly 20 times the star's photospheric flux at this wavelength, and with fractional infrared luminosity $1.4 \times 10^{-4}$ (Koerner et al. 2010). It later appeared extremely well resolved in visible scattered light with the Hubble Space Telescope's Space Telescope Imaging Spectrograph (HST/STIS), and showed a cleared central zone approximately $6^{\prime \prime}$ in radius, a sharp inner edge, while extending outwards to at least $9^{\prime \prime}$ in radius. Most importantly, the ring was found to be eccentric: it was found to extend further from the star on its southeast ansa than to the northwest one, that is, the star was found offset from the projected ring center (Krist et al. 2012; Schneider et al. 2016).

Extrasolar debris disks have been found to bear imprints of interactions with planets, as often revealed by asymmetries in their spatial distribution (Wyatt 1999; Krivov 2010). With its sharp inner edge and eccentric shape, the morphology of the debris disk of HD 202628 is analogous to that hosted by the mature yet younger ( $440 \mathrm{Myr}$, Mamajek 2012) A-type star Fomalhaut (Kalas et al. 2005), around which the presence of a 


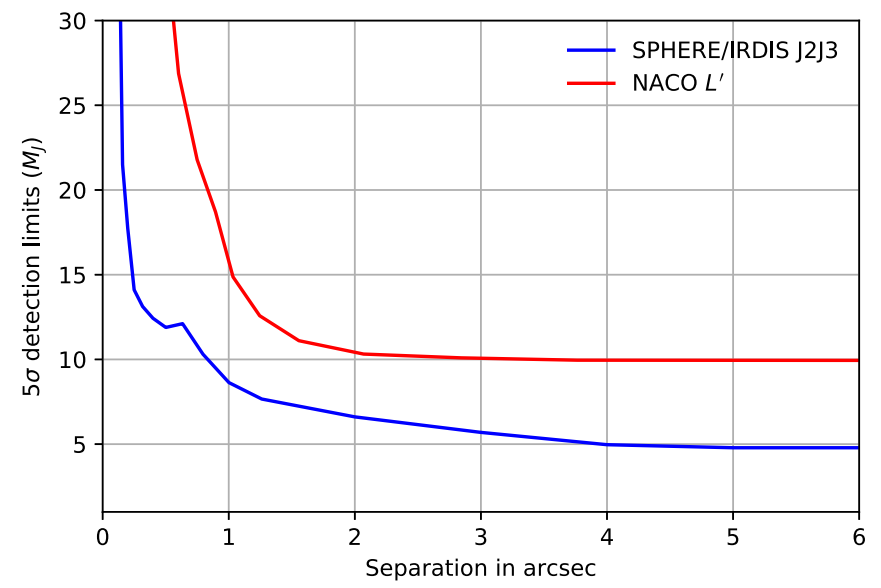

Figure 1. Five sigmas detection limits in mass reached by Irdis and $\mathrm{NaCo}$ around HD 202628, as a function of the separation to the host star, assuming an age of 1.1 Gyr and using the COND models of Baraffe et al. (2003).

planet on an eccentric orbit at several tens of astronomical unit, and carving the inner edge of the ring has been subsequently inferred through dynamical modeling (Quillen 2006; Chiang et al. 2009). Consequently, a similar perturber has been postulated around the Gyr-old solar-type star HD 202628 (Krist et al. 2012).

Dynamical modeling of debris disk asymmetries and structures allows us to predict the presence of yet undetected planetary components (Moro-Martin 2013). This approach permits us to constrain their mass and orbital properties, and hence constitutes a powerful indirect detection technique, which in addition, probes regions of exoplanet mass and orbital radius parameter space inaccessible to other methods. Indeed, it is particularly suited for systems such as Fomalhaut or HD 202628, where planets are too distant from their host star to have been detected through usual detection techniques, such as radial velocities or transits, which are biased toward shortperiod objects.

While direct imaging techniques and instruments may overcome these limitations, in mature systems such as HD 202628, planets are expected to have lost too much of their intrinsic luminosity to be detected. Around a star as old as HD 202628 , and even with the best configuration possible to probe very cold companions (for example, see the Very Large Telescope's Spectro-Polarimetric High-Contrast Exoplanet Research (VLT/SPHERE) observations of GJ 504A in dual band imaging J2J3; Bonnefoy et al. 2018), the high contrast between such companions and HD 202628 would make it difficult to directly observe objects less massive than $5 M_{\text {Jup }}$ at the inferred distance (see Figure 1). Note that current direct imaging constraints for this system obtained with VLT/NaCo (NAOS-CONICA; Nasmyth Adaptive Optics System (NAOS) Near-Infrared Imager and Spectrograph (CONICA)) in $L$ band allow us to exclude the presence of a body more massive than $\sim 50 M_{\text {Jup }}$ beyond $\sim 1.5$ (D. Mawet 2019, private communication).

Based on the geometrical constraints provided by $H S T$ for the debris disk of HD 202628, several predictions have been made on the putative eccentric belt-shaping perturber at play in this system. Applying their equation that links the width of a debris ring as seen in scattered light to the maximum mass and minimum semimajor axis of a belt-shaping perturber, Rodigas et al. (2014) theoretically derived a perturber's maximum mass of $15 M_{\text {Jup }}$ at minimum separation $\sim 1$ ". 2 . On the other hand, based on dynamical theoretical constraints for an eccentric perturber to secularly shape a ring into an eccentric ring and carving its inner edge, Pearce \& Wyatt (2014) provided a lower mass limit of $0.2 M_{\text {Jup }}$, while further extensive numerical exploration and fit to $H S T$ observations allowed Thilliez \& Maddison (2016) to find a best fit of $3 M_{\text {Jup }}$ for this eccentric companion, which means this perturber should have a mass well below the current achievable detection limits quoted above. This makes it tough to characterize it other than by carrying out dynamical modeling of the spatial structure of the ring, and thus characterizing it via its gravitational imprint on the debris disk. The aforementioned constraints were derived using the star's distance as provided by Hipparcos $(d=24.4 \mathrm{pc}$, van Leeuwen 2007) which was recently corrected by Gaia measurements, though the difference between the two values is relatively small and will therefore not affect the constraints significantly. However, the constraints were also based on the geometry of the debris disk as found by HST observations: small micron-sized particles revealed in scattered light with $H S T$ can drift far from their source due to the influence of collisions and radiation pressure (Thébault \& Augereau 2007), which leads structures seen in scattered light to be altered versions of the true dynamical structure imposed by an unseen planet (Thebault et al. 2012).

Knowledge of the debris disk geometry should be clearly improved thanks to far-IR and up to millimeter wavelengths observations, as those probe a different physical regime than visible light: the large mm-sized particles traced by these emissions tend to remain near the site of their initial collisional formation. This means that millimeter observations of a debris disk provide better constraints on the gravitational imprint of the planetary components shaping them. In addition, comparison of a debris disk morphology and content across different wavelengths provides key information on the dust population, and in particular, its size distribution (Ricci et al. 2012). This is of prime importance in the case of the HD 202628 system as in situ formation of the belt-shaping perturber has been suggested to be possible, and promoted by a steeper grain size distribution than usually expected in debris disks (Kenyon \& Bromley 2015).

We present here a whole new set of observations of the debris disk of HD 202628, which we will describe in Section 2, and analyze in Section 3: Atacama Large Millimeter/ submillimeter Array (ALMA) resolved observations at 1.3 $\mathrm{mm}$ which will allow us to significantly refine our knowledge of the parent ring geometry while probing the disk gas content, Herschel/PACS (Photodetector Array Camera and Spectrometer) and SPIRE (Spectral and Photometric Imaging Receiver) observations from 70 to $500 \mu \mathrm{m}$, which will provide photometric measurements and allow us, in combination with our ALMA data, to determine the grain size distribution of the debris disk and conclude on the possible in situ formation of distant planets around HD 202628, and finally, deeper HST/ STIS observations in scattered light, which will provide additional information on the dust grain properties and in particular, their scattering phase function. Using the new constraints on the disk geometry provided by our observations, we will set in turn constraints on the orbital properties of the eccentric perturber inferred in this system in Section 4. In addition, we will discuss the presence of a source just interior to the ring which, if linked to the system, could be 
Table 1

Summary of our ALMA/ACA Observations at $1.3 \mathrm{~mm}$ (Band 6)

\begin{tabular}{|c|c|c|c|c|c|c|c|c|}
\hline \multirow{2}{*}{$\begin{array}{l}\text { Date }^{\mathrm{a}} \\
\text { (YYYY mm dd) }\end{array}$} & \multirow{2}{*}{$\begin{array}{l}\text { Time }^{\mathrm{a}} \\
\text { (UTC) }\end{array}$} & \multirow{2}{*}{$\begin{array}{c}\text { On Source } \\
\text { (minutes) }\end{array}$} & \multirow{2}{*}{$N_{\text {Ant. }}$} & \multirow{2}{*}{$\begin{array}{l}\text { PWV } \\
(\mathrm{mm})\end{array}$} & \multirow{2}{*}{$\begin{array}{l}\text { Elevation } \\
\quad(\mathrm{deg})\end{array}$} & \multicolumn{3}{|c|}{ Calibrators } \\
\hline & & & & & & Flux & Bandpass & Phase \\
\hline 2016 Oct 19 & 01:38:51.0 & 12.8 & 10 & $0.68-0.73$ & $54.9-57.0$ & Uranus & J0006-0623 & J2056-4714 \\
\hline 2016 Oct 21 & $01: 23: 11.2$ & 48.1 & 9 & $0.39-0.48$ & $47.5-58.2$ & Neptune & J0006-0623 & J2056-4714 \\
\hline 2016 Oct 24 & $01: 17: 29.5$ & 48.1 & 8 & $0.62-1.16$ & $46.2-57.2$ & Neptune & J0006-0623 & J2056-4714 \\
\hline 2016 Oct 27 & $00: 38: 56.5$ & 48.1 & 10 & $0.36-0.55$ & $51.0-61.3$ & Neptune & J0006-0623 & J2056-4714 \\
\hline 2016 Oct 27 & $23: 38: 25.1$ & 48.1 & 9 & $0.68-1.07$ & $60.5-68.0$ & Mars & J1924-2914 & J2056-4714 \\
\hline 2016 Oct 28 & $01: 17: 52.8$ & 48.1 & 9 & $0.63-0.70$ & $43.5-54.9$ & Neptune & J0006-0623 & J2056-4714 \\
\hline 2016 Oct 28 & $23: 36: 45.8$ & 48.1 & 10 & $0.76-1.03$ & $60.1-67.9$ & Mars & J1924-2914 & J2056-4714 \\
\hline 2016 Oct 29 & $23: 48: 46.8$ & 48.1 & 10 & $1.45-1.85$ & $57.5-66.4$ & Mars & J1924-2914 & J2056-4714 \\
\hline 2016 Oct 30 & $01: 30: 46.1$ & 48.1 & 10 & $1.08-1.26$ & $39.9-51.0$ & Uranus & J0006-0623 & J2056-4714 \\
\hline 2016 Oct 30 & $23: 30: 23.8$ & 48.1 & 10 & $0.65-0.80$ & $59.8-67.7$ & Mars & J1924-2914 & J2056-4714 \\
\hline 2016 Oct 31 & $01: 21: 14.7$ & 48.1 & 10 & $0.44-0.63$ & $40.6-52.0$ & Uranus & J0006-0623 & J2056-4714 \\
\hline 2016 Nov 1 & 00:02:10.6 & 48.1 & 10 & $0.88-0.94$ & $54.0-63.8$ & Mars & J1924-2914 & J2056-4714 \\
\hline 2016 Nov 1 & $22: 56: 59.3$ & 48.1 & 10 & $1.46-1.66$ & $63.5-69.4$ & Mars & J1924-2914 & J2056-4714 \\
\hline
\end{tabular}

Note.

${ }^{a}$ At exposure start.

Table 2

Summary of our ALMA/12 m Array Observations at $1.3 \mathrm{~mm}$ (Band 6)

\begin{tabular}{|c|c|c|c|c|c|c|c|c|}
\hline \multirow{2}{*}{$\begin{array}{l}\text { Date }^{\mathrm{a}} \\
\text { (YYYY mm dd) }\end{array}$} & \multirow{2}{*}{$\begin{array}{l}\text { Time }^{\mathrm{a}} \\
\text { (UTC) }\end{array}$} & \multirow{2}{*}{$\begin{array}{l}\text { On Source } \\
\text { (minutes) }\end{array}$} & \multirow{2}{*}{$N_{\text {Ant. }}$} & \multirow{2}{*}{$\begin{array}{l}\text { PWV } \\
(\mathrm{mm})\end{array}$} & \multirow{2}{*}{$\begin{array}{l}\text { Elevation } \\
\quad(\text { deg })\end{array}$} & \multicolumn{3}{|c|}{ Calibrators } \\
\hline & & & & & & Flux & Bandpass & Phase \\
\hline 2017 Apr 15 & $11: 34: 48.2$ & 46.2 & 42 & $1.86-2.34$ & $68.6-69.6$ & Titan & J2056-4714 & J2056-4714 \\
\hline 2017 Apr 18 & $11: 24: 08.9$ & 46.2 & 41 & $1.47-1.56$ & $68.2-69.6$ & Titan & $\mathrm{J} 2056-4714$ & J2056-4714 \\
\hline 2017 Apr 24 & $10: 04: 34.5$ & 38.9 & 39 & $0.71-0.74$ & $64.0-68.0$ & Titan & $\mathrm{J} 2258-2758$ & J2056-4714 \\
\hline 2017 Apr 25 & $11: 21: 26.5$ & 46.2 & 38 & $1.10-1.44$ & $66.5-69.5$ & J2056-4714 & $\mathrm{J} 2258-2758$ & J2056-4714 \\
\hline 2017 Apr 27 & $10: 56: 34.8$ & 46.2 & 39 & $0.27-0.28$ & $67.6-69.6$ & Titan & J2056-4714 & J2056-4714 \\
\hline 2017 Apr 28 & $08: 29: 20.3$ & 46.2 & 39 & $0.24-0.26$ & $51.0-60.0$ & J2056-4714 & J1924-2914 & J2056-4714 \\
\hline 2017 Apr 29 & $11: 08: 34.5$ & 46.2 & 39 & $0.78-0.93$ & $66.0-69.5$ & J2056-4714 & $\mathrm{J} 2258-2758$ & J2056-4714 \\
\hline
\end{tabular}

Note.

${ }^{a}$ At exposure start.

circumplanetary material orbiting the expected perturber, and show how this would allow us to fully characterize the mass and orbital properties of the perturber. Finally, we present our conclusions in Section 5.

\section{Observations}

\subsection{ALMA Band 6 Observations}

We present here ALMA observations of HD 202628 in Band $6(230 \mathrm{GHz}, 1.3 \mathrm{~mm})$, under the project 2016.1.00515.S (PI: V. Faramaz). They comprise $12 \mathrm{~m}$ array observations carried out from 2017 April 15 to 29, and observations carried out with the Atacama Compact Array (ACA) from 2016 October 19 to 2016 November 2. Our ACA and $12 \mathrm{~m}$ array data comprised 13 and 7 separate observations, respectively, for which we summarize the characteristics in Tables 1 and 2. ACA data were taken using baselines ranging from 8.9 to $48.0 \mathrm{~m}$, which corresponds to angular scales of 30 !' 2 and 5.'6, respectively, while $12 \mathrm{~m}$ array data were taken using baselines ranging from 15.1 to $460.0 \mathrm{~m}$, which in this case, corresponds to angular scales of 17 !" 8 and 0 ". 6 , respectively. Given the distance of the star $(23.8$ pc), this means that the spatial scales that were probed with the ACA ranged from 133.3 to $718.8 \mathrm{au}$, and those probed with the $12 \mathrm{~m}$ array ranged from 14.3 to $423.6 \mathrm{au}$. The spectral setup consisted of four spectral windows, each $2 \mathrm{GHz}$ wide. Three were centered on $232.5,245.5$, and $247.5 \mathrm{GHz}$, and divided into 128 channels of width $15.625 \mathrm{MHz}\left(\sim 20 \mathrm{~km} \mathrm{~s}^{-1}\right)$.

Although we did not expect primordial gas to be present in a system as old as HD 202628, we nevertheless used the fourth spectral window to probe $\mathrm{CO}$ gas, via the $J=2-1$ emission line, as these can be released from collisions among planetesimals (as for instance, in the mature systems of $\eta$ Corvi and Fomalhaut; Marino et al. 2017; Matrà et al. 2017b, respectively). Therefore, the fourth spectral window was centered on $230.5 \mathrm{GHz}$, with a large number of finer channels (4096 for the $12 \mathrm{~m}$ array observations and 3840 for the ACA observations), leading to a spectral resolution of $0.5 \mathrm{MHz}$ $\left(\sim 0.6 \mathrm{~km} \mathrm{~s}^{-1}\right)$. The total time on source was $9.8 \mathrm{hr}$ with the ACA and $5.3 \mathrm{hr}$ with the $12 \mathrm{~m}$ array.

The spectral window covering the $\mathrm{CO} J=2-1$ transition $(230.538 \mathrm{GHz}$, with a spectral channel width of $488.24 \mathrm{kHz}$, or $0.63 \mathrm{~km} \mathrm{~s}^{-1}$ ) was extracted from each of the $12 \mathrm{~m}$ array observations, and combined to produce a visibility data set for $\mathrm{CO}$ imaging. We subtracted continuum emission from the visibilities using the uvcontsub task in CASA v5.1.0. Then, we produced a dirty image of the $\mathrm{CO}$ data set using the tclean CASA task, with natural weighting. We find no clear emission at or near the radial velocity of the star in the data cube, which has an rms sensitivity of $0.36 \mathrm{mJy}^{-1}$ beam $^{-1}$ in a $0.63 \mathrm{~km} \mathrm{~s}^{-1}$ channel, for a synthesized beam size of $0 . " 96 \times 0$." 77 . 
Table 3

List of HST HD 202628 Observations

\begin{tabular}{|c|c|c|c|c|c|c|c|}
\hline $\begin{array}{l}\text { Date } \\
\text { YYYY MM DD }\end{array}$ & $\begin{array}{c}H S T \\
\text { Dataset }\end{array}$ & $\begin{array}{c}\text { Total } \\
\text { Exposure } \\
\text { Time }\end{array}$ & $\begin{array}{c}\text { Number of } \\
\text { Subexposures }\end{array}$ & $\begin{array}{l}\text { CCD } \\
\text { Gain }\end{array}$ & Occulter & Orientation $^{\mathrm{a}}$ & $\begin{array}{l}\text { Program } \\
\text { Number }\end{array}$ \\
\hline 2011 May 15 & OBHS05010 & $2256 \mathrm{~s}$ & 8 & 1 & WEDGEA1.8 & $-165^{\circ} .0$ & 12291 \\
\hline 2011 May 15 & OBHS06010 & $2256 \mathrm{~s}$ & 8 & 1 & WEDGEA1.8 & $-137^{\circ} .0$ & 12291 \\
\hline 2014 May 2 & OC8F01010 & $1976 \mathrm{~s}$ & 8 & 1 & WEDGEA1.8 & -160.5 & 13455 \\
\hline 2014 May 2 & OC8F02010 & $1976 \mathrm{~s}$ & 8 & 1 & WEDGEA1.8 & -145.5 & 13455 \\
\hline 2014 May 2 & OC8F03010 & $1976 \mathrm{~s}$ & 8 & 1 & WEDGEA1.8 & -130.5 & 13455 \\
\hline 2014 Jul 12 & OC8F04010 & $1976 \mathrm{~s}$ & 8 & 1 & WEDGEA 1.8 & -115.5 & 13455 \\
\hline 2014 Jul 12 & OC8F05010 & $1976 \mathrm{~s}$ & 8 & 1 & WEDGEA1.8 & -100.5 & 13455 \\
\hline 2014 Jul 12 & OC8F06010 & $1976 \mathrm{~s}$ & 8 & 1 & WEDGEA1.8 & -85.5 & 13455 \\
\hline 2014 Aug 7 & OC8F07010 & $1976 \mathrm{~s}$ & 8 & 1 & WEDGEA1.8 & -70.5 & 13455 \\
\hline 2014 Aug 7 & OC8F08010 & $1976 \mathrm{~s}$ & 8 & 1 & WEDGEA1.8 & $-55^{\circ} .5$ & 13455 \\
\hline 2014 Aug 7 & OC8F09010 & $1976 \mathrm{~s}$ & 8 & 1 & WEDGEA1.8 & -40.5 & 13455 \\
\hline 2015 Sep 17 & OCJC01040 & $1630 \mathrm{~s}$ & 5 & 4 & WEDGEA1.0 & -18.0 & 13786 \\
\hline 2015 Sep 17 & OCJC02040 & $1630 \mathrm{~s}$ & 5 & 4 & WEDGEA1.0 & 1.6 & 13786 \\
\hline 2015 Sep 17 & OCJC04040 & $1630 \mathrm{~s}$ & 5 & 4 & WEDGEA1.0 & 21.0 & 13786 \\
\hline 2015 May 30 & OCJC 05040 & $1630 \mathrm{~s}$ & 5 & 4 & WEDGEA1.0 & $-117^{\circ} 0$ & 13786 \\
\hline 2015 May 30 & OCJC06040 & $1630 \mathrm{~s}$ & 5 & 4 & WEDGEA1.0 & -140.0 & 13786 \\
\hline 2015 May 30 & OCJC08040 & $1630 \mathrm{~s}$ & 5 & 4 & WEDGEA1.0 & -163.0 & 13786 \\
\hline
\end{tabular}

Note.

${ }^{\text {a }}$ Angle from north through east to the $+Y$ image axis as reported by the ORIENTAT image file header keyword.

\subsection{Herschel/PACS and SPIRE Observations}

Herschel/PACS observations using the mini scan map AOT (Astronomical Observing Template) took place on 2012 March 28. Ten scan legs with length $3^{\prime}$ were executed 3 times, repeated in two concatenated AORs (Astronomical Observation Request) scanning along the PACS array diagonal of $70^{\circ}$ and $110^{\circ}$. This sequence was done once for simultaneous 70 and $160 \mu \mathrm{m}$ observations, and a second time for simultaneous 100 and $160 \mu \mathrm{m}$ observations. The scans were filtered to remove pattern noise, excluding the region around the target, and rendered into mosaics with 1. ! 0,1 ." 0 , and 2 !" 0 pixels (at 70 , $100,160 \mu \mathrm{m})$ using customized routines in Version 10 of the Herschel Interactive Processing Environment (HIPE) software package. Herschel/SPIRE observations took place on 2012 May 11 using five repetitions of the small map AOT in each of the three photometric bands. Processed mosaics with $6{ }^{\prime \prime}, 10^{\prime \prime}$, and $14^{\prime \prime}$ pixels (at $250,350,500 \mu \mathrm{m}$ ) were retrieved from the Herschel Archive.

\subsection{New HST/STIS Observations}

HD 202628 has been observed with the HST/STIS coronagraph $\left(0\right.$ ". 05 pixel $\left.^{-1}\right)$ in three separate Guest Observer (GO) programs (Table 3). The first, GO-12291 (PI = Krist), was a 20-orbit imaging survey of 10 stars with infrared excesses measured by the Spitzer Space Telescope. Each target was observed over two consecutive orbits, with the telescope rolled about the star by $28^{\circ}$. Of those, a disk was seen only around HD 202628 (Krist et al. 2012). The extreme faintness of the disk in the 2011 data meant that additional integrations were needed to better define its morphology. A follow-up program, GO-13455 (PI = Krist), obtained 9 more orbits of images at 9 different orientations spread over three epochs in 2014, which are described here for the first time. Finally, a separate program that revisited previously imaged debris disks (GO-13786, PI = Schneider) obtained 6 more orbits of data at 6 orientations at two epochs in 2015 (Schneider et al. 2016).
Due to the large brightness difference between a star and its debris disk, the surface brightness of the wings of the star's instrumental point-spread function (PSF) is typically greater than the disk's. A coronagraph is used to suppress the diffraction pattern caused by the telescope's obscurations; a deformable mirror, if present, can be used to further reduce the starlight by correcting for optical aberrations that scatter stellar flux into the PSF wings (though there is not one on any HST instrument). The residual starlight is then removed from the images using some sort of post-processing technique. The simplest, reference differential imaging (RDI), is the subtraction of a reference star image from the target star's. It has been used extensively on HST and ground-based high-contrast data. Another technique is angular differential imaging (ADI), which extracts the astronomical signal (e.g., disk) by observing the field at multiple orientations and solving for what moves (the sky) and what does not (the PSF).

The STIS coronagraph uses a crossed pair of wedges to block the star at an intermediate focal plane, with the wedge position chosen to allow imaging as close to the star as desired (the wider wedge positions offer better diffraction suppression). At a subsequent pupil in the optical train, a Lyot stop mask is used to suppress diffraction from the outer edge of the telescope aperture. Because the STIS Lyot stop does not mask the telescope secondary mirror or its support spiders, diffraction spikes remain visible in the coronagraphic images and the wings of the PSF are suppressed by only a factor of a few (Krist 2004). Lacking a deformable mirror, the remaining starlight must be removed using post-processing. PSF subtraction provides the greatest gain in starlight suppression with HST due to its relatively stable (compared to the ground) PSF, rather than the suppression provided by the unoptimized coronagraph alone.

The STIS coronagraph does not have any filters, so its bandpass is effectively limited by the wavelength response of the CCD detector, which spans over 250-1100 nm. This complicates finding suitable reference PSF stars for RDI post- 
processing. The stellar diffraction pattern varies with wavelength, so small differences in the spectral energy distributions (SEDs) between the target and reference stars can lead to mismatches in the PSFs and cause residual artifacts in the processed images. The broader the bandpass, the greater the effects from SED mismatches will be, so a STIS reference star must be chosen that matches the target's star color extremely well.

In the original GO-12291 survey by Krist et al., the desire to avoid the (at a minimum) 10 additional orbits needed for the 10 matching reference stars led to the use of ADI post-processing with multi-orientation observations instead of RDI. Besides saving orbits, ADI avoids the introduction of residuals due to stellar color differences. This same technique was used to first image the disk around HD 207129 (Krist et al. 2010); the same iterative ADI algorithm used here was described in that paper. The drawback of the ADI method for an extended object like a disk is that self-subtraction will likely occur, depending on the object's morphology. For example, a face-on disk would appear to be part of the PSF since it does not appear to move with telescope orientation, so it would not appear in the processed image. For less inclined, ring-shaped disks like HD 207129 and HD 202628, the self-subtraction can be reduced by maximizing the amount of orientation change and the number of orientations.

Prior to post-processing, all of the images were retrieved from the HST archive. Although the calibration pipeline combines, with cosmic-ray rejection, the flat-fielded subexposures (_flt.fits) into final images (_crj.fits), no image registration is done. For the best post-processing results, however, this is needed, especially since the PSF structure has high-spatialfrequency streaks. By subtracting one frame from another the residuals caused by drift of the star within an orbit can be seen as an oversubtraction on one side of the star and undersubtraction on the other, especially in the diffraction spikes. Using cubic convolution interpolation, each subexposure was iteratively shifted by subpixel amounts and subtracted from the first 2011 subexposure until the residuals appeared to be visually minimized. Shifts as low as 0.02 pixels (1 mas) produced noticeable results. The aligned subexposures for each orientation were then combined with cosmic ray rejection.

The image sets from 2011, 2014, and 2015 were separately ADI processed (for consistency, the 2011 data were reprocessed). This avoided the long-term changes in the PSF. Also, the 2015 data were taken at a different wedge position than the 2011 and 2014 sets, and the PSF is different for each position. Note that the ADI processing of the 2015 data and the derived results presented here are independent of those for the same data discussed by Schneider et al. (2016), who used RDI instead. The center of rotation in the aligned images was determined by fitting for the intersection of the diffraction spikes. The combination of these three images are used hereafter, representing a total of $8.9 \mathrm{hr}$ of integration. An evaluation of the impact of disk self-subtraction due to ADI processing using a disk model is given in the Appendix B. As will be shown, there is no significant effect that would alter the observed morphology of the disk.

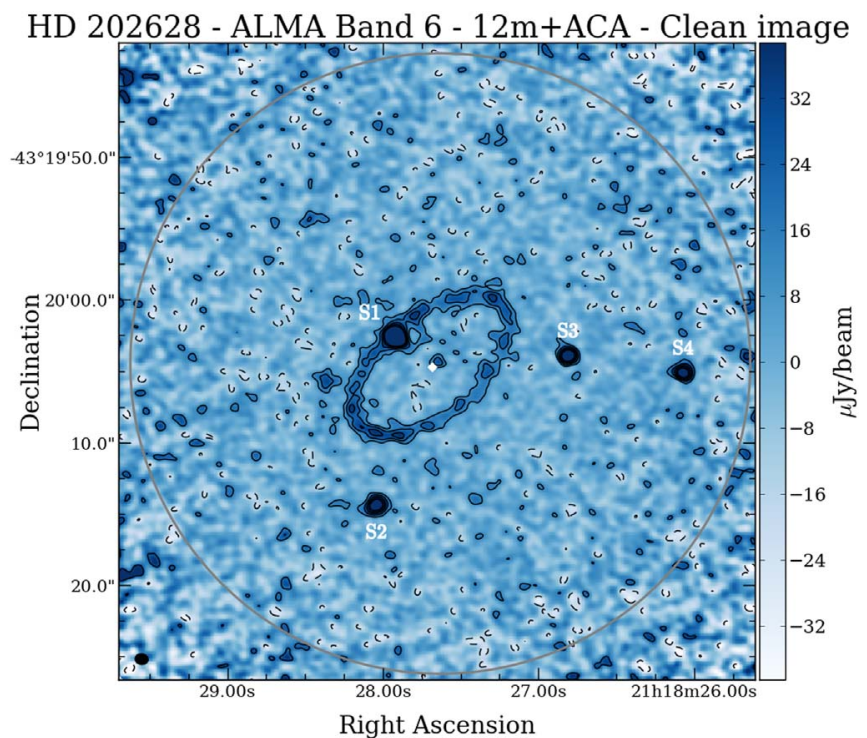

Figure 2. ALMA 1.3 continuum observations of HD 202628, combining the $12 \mathrm{~m}$ and ACA data. Contours show the $\pm 2,4,6, \ldots \sigma$ significance levels, with $\sigma=5.2 \mu \mathrm{Jy}_{\text {beam }^{-1}}$. The synthesized beam, shown on the lower left side of the image, has dimensions $0.92 \times 0.75$, with position angle $83^{\circ}$. The stellar photosphere appears inner to the ring and is detected with $\mathrm{S} / \mathrm{N}$ of nearly 6 , and the ring center of symmetry (white diamond) is slightly offset from it. Bright sources $(\mathrm{S} / \mathrm{N}>6)$ within the field are labeled from $\mathrm{S} 1$ to $\mathrm{S} 4$. The gray circle indicates the $50 \%$ response of the ACA primary beam and the color bar shows the fluxes in $\mu \mathrm{Jy}$ beam $^{-1}$

\section{Results}

\subsection{ALMA Band 6 Observations}

Calibrations were applied using the pipeline provided by ALMA. We used the TCLEAN algorithm in CASA (Common Astronomy Software Applications) version 5.1.1 (McMullin et al. 2007) to perform the image reconstruction of the continuum emission, that is, to obtain the inverse Fourier transform of the observed visibilities. We combined the four spectral windows in order to recover the maximum signal-tonoise ratio $(\mathrm{S} / \mathrm{N})$ and used a mask generated with our best-fit model (see Section 3.1.3). We used a natural weighting scheme, a cell size of 0 " 08 , and an image size of $800 \times 800$ pixels in order to cover the primary beam, as well as a mask generated with our disk best-fit model (see Section 3.1.3). The resulting synthesized beam has dimensions 0 ". $92 \times 0$ ". 75 , with position angle (PA) $83^{\circ}$. The rms was measured in a large region far from the sources present in the field of view, and was found to be $\sigma=5.2 \mu \mathrm{Jy}_{\text {beam }^{-1}}$. We further corrected the image for the primary beam, and show the results in Figure 2.

The ring appears clearly defined in our image, as well as the star, that is visibly offset from the ring center of symmetry. In addition, several bright sources appear within the field, one of them being superimposed on the ring. In the following, we give quantitative measurements for all these components: ring's photometry and geometry, stellar photospheric flux and position, bright sources' photometry and position, and finally gas content.

\subsubsection{The Star}

The peak emission of the unresolved stellar photosphere at $1.3 \mathrm{~mm}$ is found to be $29.3 \pm 5.2 \mu \mathrm{Jy} \mathrm{beam}^{-1}$. This is higher than our prediction of a star's peak flux emission of $19 \mu \mathrm{Jy}_{\text {beam }^{-1}}$, which was derived by fitting a Kurucz model 
Table 4

Characteristics of the Bright Sources Within the Field of View

\begin{tabular}{|c|c|c|c|c|c|c|c|}
\hline \multirow{2}{*}{ Source } & \multirow{2}{*}{$\begin{array}{l}\text { Integrated } \\
\text { Flux }(\mu \mathrm{Jy})\end{array}$} & \multirow{2}{*}{$\begin{array}{c}\text { Peak } \\
\left(\mu \mathrm{Jy} / \text { beam }^{-1}\right)\end{array}$} & \multicolumn{2}{|c|}{ Position $^{\mathrm{a}}$} & \multicolumn{3}{|c|}{ Deconvolved FWHM Axes } \\
\hline & & & R.A. (s) & Decl. (") & Major (mas) & Minor (mas) & $\mathrm{PA}\left({ }^{\circ}\right)$ \\
\hline $\mathrm{S} 1$ & $285 \pm 18$ & $189.0 \pm 7.6$ & $21: 18: 27.905 \pm 0.002$ & $-43.20 .02 .629 \pm 0.017$ & $700 \pm 108$ & $460 \pm 153$ & $28 \pm 22$ \\
\hline S3 & $123 \pm 10$ & $100.0 \pm 5.0$ & $21: 18: 26.798 \pm 0.002$ & $-43.20 .03 .896 \pm 0.018$ & $456 \pm 139$ & $299 \pm 294$ & $8 \pm 77$ \\
\hline S4 & $150 \pm 11$ & $114.7 \pm 5.4$ & $21: 18: 26.059 \pm 0.002$ & $-43.20 .05 .077 \pm 0.017$ & $514 \pm 139$ & $405 \pm 194$ & $52 \pm 63$ \\
\hline
\end{tabular}

Note. These measurements have been made using an elliptical Gaussian fit to the sources.

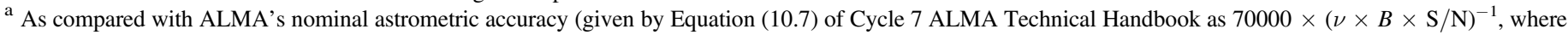

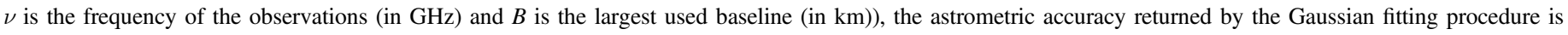
consistent for the sources observed at the largest S/N (that is, S1 and S4), while it is smaller by a factor of two for the sources S2 and S3.

photosphere to the stellar photometry between 1 and $24 \mu \mathrm{m}$ (see Section 3.2.2). With $\sigma=5.2 \mu \mathrm{Jy}_{\text {beam }}{ }^{-1}$, HD 202628 is detected with a $\mathrm{S} / \mathrm{N}$ of nearly 6 , which allows us to constrain its position. A Gaussian ellipse fitted to the photosphere's emission is centered on $21^{\mathrm{h}} 18^{\mathrm{m}} 27^{\mathrm{s}} .653 \pm 0.004$ in R.A., and $-43^{\circ} 20^{\prime} 04$ ". $267 \pm 0.04$ in decl. ${ }^{14}$

Being able to detect the star's emission and constrain its position is crucial in order to determine the geometry of the debris ring. Since the debris ring has been found to be eccentric in HST data, its center of symmetry was expected to be offset from the star, as is already visible in our image. Knowledge of the star position allows us to measure this offset and retrieve constraints on the ring eccentricity (see Section 3.1.3).

\subsubsection{Bright Sources}

Apart from the disk and the star, the field contains four additional bright sources, for which we retrieve the extent, brightness, and position. For each source, and within the CASA viewer, we performed an elliptical Gaussian fit over a rectangular region encompassing the source. This procedure returns the best fit's center, its peak and integrated fluxes, along with its convolved elliptical dimensions and position angle. If the source is found resolved, unconvolved ellipse characteristics are returned as well by the fit. We summarize this information in Table 4. The brightest source (labeled S1 in Figure 2), appears within the ring, northeast to the star, while the other three sources (labeled S2, S3, and S4) are exterior to the ring. Note that we used an image from which our disk bestfit model (see Section 3.1.3) was subtracted, so as to disentangle the emission of the source S1 from that of the ring. All the sources were marginally resolved and, with $\sigma=5.2 \mu \mathrm{Jy} / \mathrm{beam}^{-1}$, were found to have $\mathrm{S} / \mathrm{Ns} 36,17,19$, and 22, fro $\mathrm{S} 1, \mathrm{~S} 2, \mathrm{~S} 3$, and $\mathrm{S} 4$ respectively.

\subsubsection{The Ring}

As compared to the HST images of Krist et al. (2012) and Schneider et al. (2016), the debris disk appears as a narrow ring $\left(\sim 1^{\prime \prime}\right.$ wide) with ALMA. It is contained within two projected ellipses of dimensions $\sim 3^{\prime \prime} \times \sim 6^{\prime \prime}$ and $\sim 4^{\prime \prime} \times \sim 7^{\prime \prime}$, both with position angle of $\sim-50^{\circ}$.

\footnotetext{
${ }^{14}$ Note that the astrometric accuracy returned by the Gaussian fitting procedure is a factor of 2-3 smaller than ALMA's nominal astrometric accuracy given by $70000 \times(\nu \times B \times \mathrm{S} / \mathrm{N})^{-1}$, where $\nu$ is the frequency of the observations (in $\mathrm{GHz}$ ) and $B$ is the largest used baseline (in $\mathrm{km}$ ) (Equation (10.7) of Cycle 7 ALMA Technical Handbook).
}

More importantly, their center of symmetry is offset by $\sim 0$. 5 from the star, which confirms the ring is intrisically eccentric.

In order to further characterize the ring's geometry, orientation, and mass, we used the Python emcee procedure (Foreman-Mackey et al. 2013), which allows us to perform a Markov Chain Monte Carlo (MCMC) fit of a debris disk model emission to our observations. The model disk emission is computed thanks to the ray-tracing disk code first developed by Rosenfeld et al. (2013) and Flaherty et al. (2015) to model protoplanetary disks emission, and later modified to model debris disks emission, which are optically thin in comparison with optically thick protoplanetary disks (Daley et al. 2019). ${ }^{15}$

The dust temperature is derived assuming blackbody grains irradiated by the star, while its opacity is set to $2.3 \mathrm{~cm}^{2} \mathrm{~g}^{-1}$ (Beckwith et al. 1990; Andrews \& Williams 2005; Andrews et al. 2013). This opacity has been originally derived from an empirical power-law inspired by dust grain distribution models, and hence there is an assumption on the dust composition and grain size distribution underlying it. However, it shall be noted that there remains a factor of five uncertainty on the opacity normalization, and more importantly, that the dust grain size distribution does not extend up to the parent planetesimal population of the debris disk. Consequently, the mass derived by the radiative transfer code corresponds to the mass of the grains seen at the wavelength of the observations $(\sim 1 \mathrm{~mm})$, and the parent bodies could have much more mass.

We used a circular ring model, which treated the offset of its center of symmetry from the center of our image with two free parameters (R.A. and decl.). From the original HST data, the inferred eccentricity of the ring is close to 0.2 (Krist et al. 2012). Since the semiminor axis $b$ of an ellipse is by definition $b=a \sqrt{1-e^{2}}$, where $a$ is its semimajor axis and $e$ its eccentricity, then the difference between the major and minor axis is expected to be $\Delta=a-b=a\left(1-\sqrt{1-e^{2}}\right)$, that is, $\sim 2 \%$ of the semimajor axis. Using the largest extent of the ring of $\sim 250$ au observed with HST as a conservative value, then the minor axis is expected to be $\lesssim 0$ "' 2 smaller than the major axis. This is four times smaller than the beam of our ALMA observations, and therefore, is not detectable in our images. This means that with ALMA observations of the ring only, one would not be able to disentangle a projected circular ring from a ring of eccentricity 0.2 .

On the other hand, the deprojected offset between the star and the ring center of symmetry is $a e$, that is, it is expected to

\footnotetext{
15 This code is publicly available and can be found here: https://github.com/ kevin-flaherty/disk_model.
} 
Table 5

Result of the MCMC Fit on the Ring Geometry

\begin{tabular}{lc}
\hline \hline Parameter & Value \\
\hline$M_{\text {disk }}\left(M_{\oplus}\right)$ & $(1.36 \pm 0.06) \times 10^{-2}$ \\
Inner radius (au) & $143.1 \pm 1.7$ \\
Outer radius (au) & $165.5 \pm 1.4$ \\
Inclination $\left({ }^{\circ}\right)$ & $57.4 \pm 0.4$ \\
PA $\left(^{\circ}\right)$ & $-50.4_{-0.5}^{+0.4}$ \\
Eccentricity & $0.09_{-0.01}^{+0.02}$ \\
\hline
\end{tabular}

Note. Error bars correspond to the 16-84 percentiles values. The mass represents the mass in solids of size comparable to the wavelength of the observations ( $\sim 1 \mathrm{~mm}$ ), and is thus a lower limit on the mass of the debris disk.

be one order of magnitude higher than the difference between the semimajor and semiminor axis. Indeed, the projected offset as measured in HST observations is $\sim 0$." 8 . This is precisely the size of our ALMA beam, and therefore, the eccentricity of the ring can be retrieved by assessing the position of the star, and measuring its offset from the center of symmetry of a circular ring model. Note that we have found that the star is not strictly at the center of our image, and therefore, a correction will be applied to the offset found in our MCMC run to retrieve the offset from the star.

The other free parameters of the disk are: its mass, inner radius, outer radius, inclination, and position angle, for a total of 7 free parameters. It is assumed to have a constant surface density. While the disk geometry derived via MCMC is dependent on accurate modeling of the surface brightness distribution, the ring is only barely resolved in width, and variations of the surface density are not expected to be significant in this case, hence our choice of a constant surface density. Since the emission from the source S1 overlaps that of the ring, we include a Gaussian source in our model, allowing for its peak brightness, width, and offset from the image center in R.A. and decl. to vary. We included as well the sources S2S4 in our model as Gaussian emissions. The total number of free parameters in our model is therefore 23. In the frame of a MCMC fitting procedure, these 23 parameters are allowed to vary, and the corresponding model disk emission for each parameter set tested was Fourier transformed into visibilities for comparison with our ALMA data. We summarize our findings in Table 5. In Figure 3, we show our best-fit model, along with the residuals once the model is subtracted from our observations.

In order to characterize further the geometry of the ring, and in particular to translate its offset from the star into an intrinsic eccentricity, we use the fact that the center of the ellipse, its focus, and its periastron, remain aligned in the projected ellipse. The ring's true eccentricity is given by the ratio between the deprojected offset $a e$ and the ring deprojected semimajor axis $a$, however, since these two quantities are reduced by the same factor during the projection because they share the same direction, the ring's true eccentricity is thus also equal to the ratio between the projected offset and the projected semimajor axis.

We find that the projected offset of the ring center of symmetry from the star is $0.155_{-0 / 1}^{+0 / 1}$, while the ring's inner edge projected semimajor axis is $5 . \prime 9 \pm 0$ ! 1 , which leads the ring's true eccentricity to be $0.09 \pm 0.02$. In addition, the pericenter is found to have a position angle of $318^{\circ} \pm 10^{\circ}$ and the apocenter $138^{\circ} \pm 10^{\circ}$.
Note that the value of $0.09 \pm 0.02$ for the ring eccentricity is half the one found by Krist et al. (2012), but that the orientation and eccentricity of the 2012 image was derived from purely visual ellipse fits to the inner apparent edge of the ring, which was not very well defined (and still is not even in our much deeper data). Similarly, independent visual fits to the new, combined HST yields an eccentricity of $0.14 \pm 0.02$ with an inclination of $56^{\circ} .7 \pm 1.5$ and major axis PA of $-52^{\circ} .9 \pm 1^{\circ} .3$. While the inclination and PA of the disk are compatible across both ALMA and HST data sets, a small discrepancy remains on the eccentricity, which is likely due to the difficulty in defining the inner edge of the diffuse ring in HST observations.

Finally, using our best-fit model of the sources, we subtracted them to produce an image of the ring in which the source $\mathrm{S} 1$ in particular would be disentangled from the ring. This allows us to determine the total flux of the debris ring, which, as delimited by its $2 \sigma$ contour, is found to be $959 \pm 96 \mu \mathrm{Jy}$ (including a 10\% absolute flux uncertainty).

Note that in Figure 2, our ALMA observations seem to show an emission enhancement in the SE direction, that is, in the direction of the apocenter. This would be expected from an eccentric debris disk, which is bound to be intrinsically asymmetric in azimuth, with the apocenter region being denser than the pericenter region, leading to a so-called "apocenterglow" at millimeter wavelengths (Pan et al. 2016). However, while this phenomenon should be expected from an eccentric ring such as the one of HD 202628, our observations lack the sensitivity for us to be formally conclusive. This is confirmed as our best-fit model is that of an azimuthally uniform ring, and that no residuals remain at apastron. Hence the detection of the apocenter-glow remains qualitative.

\subsubsection{Gas Content}

Although no clear CO $J=2-1$ emission is observed in the datacube, we use the spectro-spatial filtering technique of Matrà et al. 2015, 2017b to boost the $\mathrm{S} / \mathrm{N}$ of any line emission that may be present but diluted over many spatial and spectral resolution elements. In order to do so, we proceed with the assumption that any gas present is co-located with the belt's continuum emission and moving at Keplerian velocity around the central star, as this has been the case in the vast majority of gas-bearing debris disks observed so far.

We begin by shifting the spectra in each of the cube's pixels by the negative of the Keplerian velocity expected at that belt location. To calculate the Keplerian velocity field, we use the best-fit longitude of ascending node, argument of pericenter and inclination to the line of sight inferred from continuum emission, and a stellar mass of $1 M_{\odot}$ (see Appendix A). We try positive and negative inclinations as its sign (or similarly, the rotation direction) is unknown, and this determines whether either the NW or the SE ansa is approaching Earth.

Then, we integrate emission spatially over a $\sim 1^{\prime \prime}$-wide elliptical mask covering the region where the ring's continuum emission is detected at a $>2 \sigma$ level. This leads to a spectrospatially filtered 1D spectrum for each sign of the inclination, with rms noise levels of $2.3 \mathrm{mJy}$ for the native channel size of $0.63 \mathrm{~km} \mathrm{~s}^{-1}$. No significant emission is seen in either spectra at the radial velocity of the central star $\left(12.0 \pm 0.2 \mathrm{~km} \mathrm{~s}^{-1}\right.$ in the barycentric frame, Gaia Collaboration et al. 2018). We therefore set a $3 \sigma$ upper limit on the integrated CO $J=2-1$ line flux within the HD 202628 belt of $12 \mathrm{mJy} \mathrm{km} \mathrm{s}{ }^{-1}$, assuming the spectro-spatially filtered line is unresolved (as 
(a)

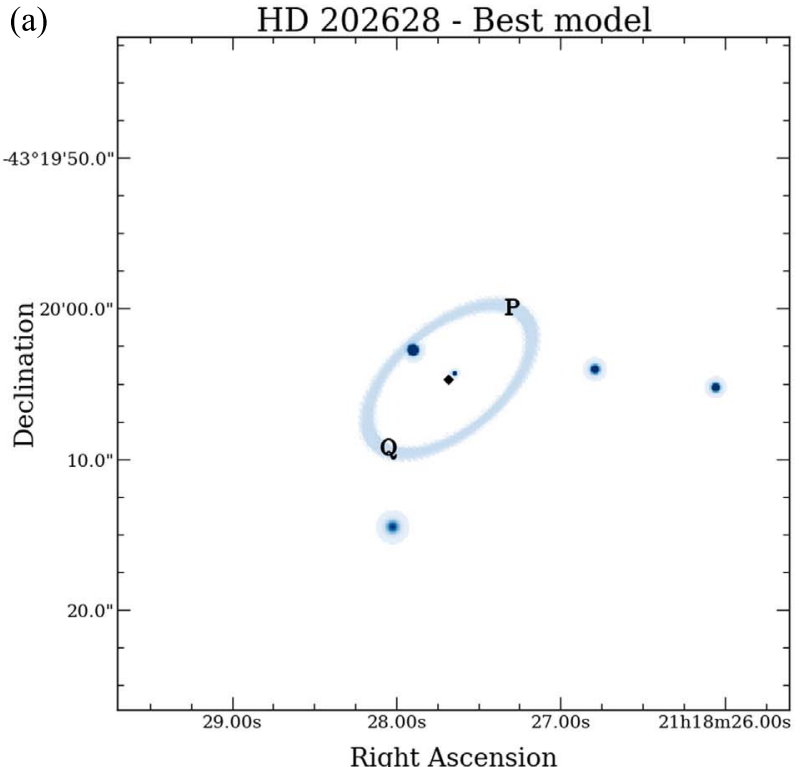

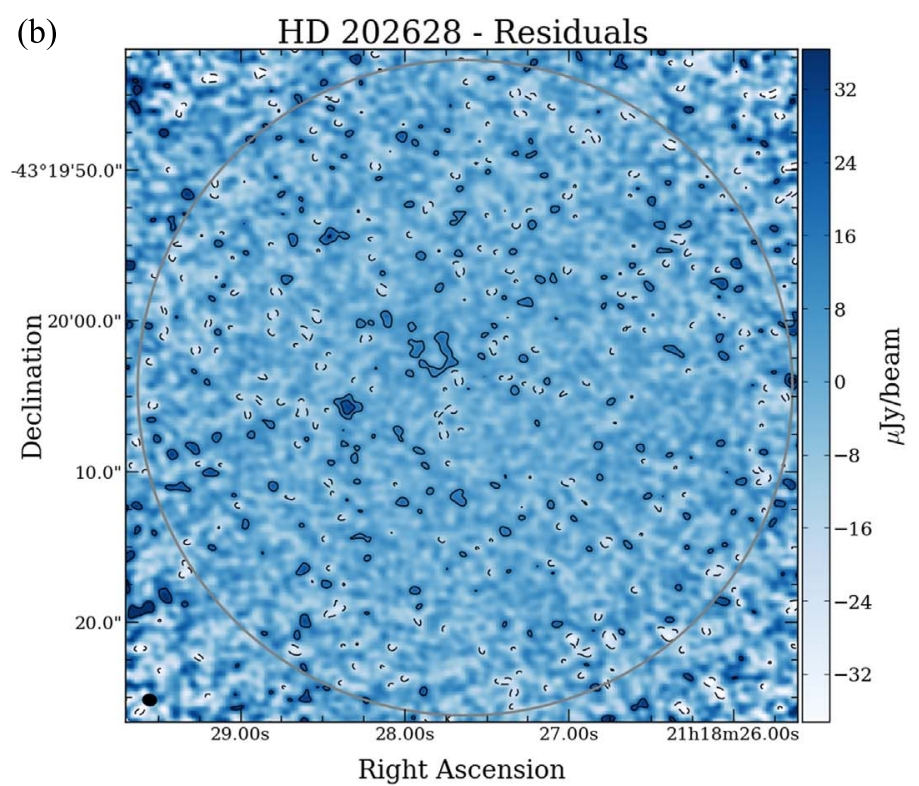

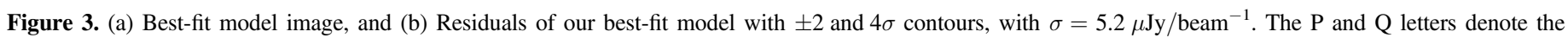
pericenter and apocenter of the ring, respectively, while the diamond shows the ring center of symmetry.

would be expected, see e.g., Matrà et al. 2017b), and adding a $10 \%$ flux calibration uncertainty in quadrature.

To obtain an upper limit on the total CO mass, we assume optically thin emission and calculate $\mathrm{CO}$ rotational excitation using the non-LTE (Local Thermodynamic Equilibrium) code of Matrà et al. (2015), including the effect of UV/IR fluorescence (Matrà et al. 2018). To calculate excitation of electronic (UV) and vibrational (IR) levels leading to fluorescent excitation, we use the interstellar radiation field (ISRF) from Draine (1978) with the long-wavelength addition of van Dishoeck et al. (2006), superimposed to a PHOENIX photospheric model fitted to observed stellar fluxes $\left(T_{\text {eff }}=5780 \mathrm{~K}, \log (g)=3.6,[\mathrm{M} / \mathrm{H}]=-0.1\right)$, and rescaled to represent the flux received at the ring location.

We explore the entire parameter space of density of collisional partners (here assumed to be electrons, but note that this does not affect the result, see Matrà et al. 2015), and kinetic temperatures between 10 and $250 \mathrm{~K}$. This leads to a range of $3 \sigma \mathrm{CO}$ mass upper limits between 1.4 and $26 \times 10^{-7} M_{\oplus}$. This is four to five orders of magnitude smaller than the disk dust mass (see Table 5). Consequently, the condition for a narrow ring to be formed as a result of interactions between solids and gas stated by Lyra \& Kuchner (2013), namely a dust-to-gas mass ratio smaller than 1, is not fulfilled, and we can therefore discard this scenario for the debris ring of HD 202628.

A basic calculation of the column density assuming the ring is circular, face-on and uniform in azimuth leads to a value of $4 \times 10^{12} \mathrm{~cm}^{-2}$, and allows us to set an upper limit on the optical depth of 0.04 (Equation (3) of Matrà et al. 2017a). This confirms that any $\mathrm{CO}$ emission that may be present below the detection limits is optically thin at $\mathrm{mm}$ wavelengths.

Such low levels of $\mathrm{CO}$, even assuming the presence of $\mathrm{H}_{2}$ with a low $\mathrm{CO} / \mathrm{H}_{2}$ abundance ratio of $10^{-6}$, cannot be shielded over the lifetime of the system against photodissociation from ISRF UV photons (which dominate over the star at the ring's location), using shielding factors from Visser et al. (2009). That means that any $\mathrm{CO}$ that may be present will be photodissociated in $\sim 120 \mathrm{yr}$, and cannot be primordial in origin. The only possibility would then be continuous replenishment through exocometary gas release, as observed in a number of debris disks so far.

In this scenario, assuming steady state $\mathrm{CO}$ production and destruction through the collisional cascade allows us to estimate the mass fraction of $\mathrm{CO}\left(+\mathrm{CO}_{2}\right.$, given $\mathrm{CO}_{2}$ could also be rapidly photodissociated, contributing to the observed CO) ice in the belt's exocomets (see Matrà et al. 2017b, for details). For HD 202628, given its belt average radius of 155 au and width of $21.5 \mathrm{au}$, for a stellar luminosity of $1 L_{\odot}$ and mass of $1 M_{\odot}$, and a fractional luminosity of $1.4 \times 10^{-4}$, we obtain an upper limit on the $\mathrm{CO}\left(+\mathrm{CO}_{2}\right)$ mass fraction of exocomets of $36 \%-91 \%$. This is consistent or above values found in other exocometary belts and solar system comets (see Figure 6 of Matrà et al. 2017b), which indicates that it is still possible for exocomets in HD 202628's belt to have typical CO ice abundances despite the non-detection presented here.

\subsection{Hersche1/PACS and SPIRE Observations}

The Herschel image mosaics are presented in Figure 4. At $70 \mu \mathrm{m}$ the resolution is sufficient to see the central clearing and higher surface brightness on the ring's west side. Gaussian fitting finds source major axis FWHM (Full Width at Half Maximum) values of 13 " 6,15 " 4 , and 15 ". 2 at the three PACS wavelengths, after accounting for the instrumental beamsizes reported by Bocchio et al. (2016). The major axis PA is consistent with higher-resolution measurements at other wavelengths. At 100 and $160 \mu \mathrm{m}$ the source FWHM of $\sim 360$ au are consistent with each other and with the size of the ring as seen in scattered light (Krist et al. 2012; Schneider et al. 2016; and Section 2.2 below), while at $70 \mu \mathrm{m}$ the ring FWHM of 320 au is noticeably smaller, suggesting that the emission is dominated by the ring inner edge.

\subsubsection{Pericenter-glow}

A key aspect of the $70 \mu \mathrm{m}$ image is its asymmetrically bright emission peak several arcseconds to the west of the star, as shown in Figure 5. The surface brightness in this region is 

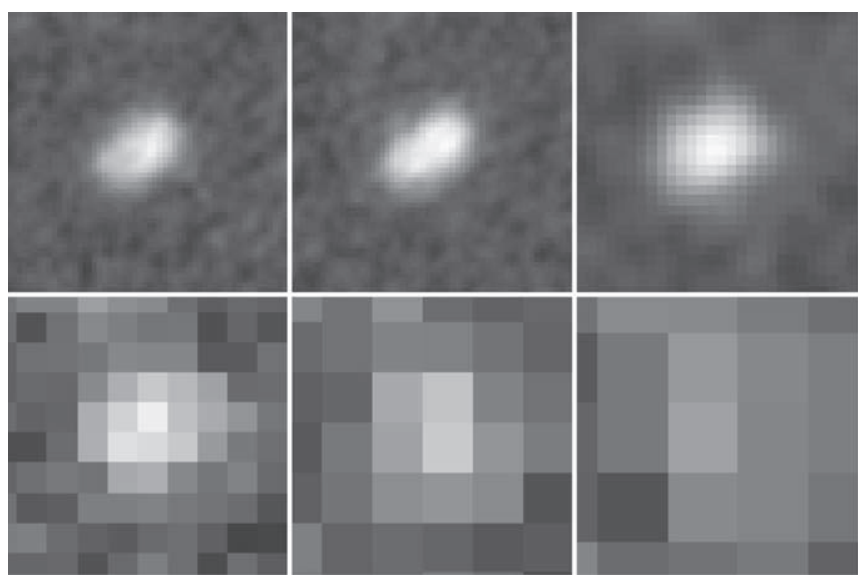

Figure 4. HD 202628 maps as seen in the 70, 100, and $160 \mu \mathrm{m}$ channels of Herschel/PACS (top row) and the 250, 350, and $500 \mu \mathrm{m}$ channels of Herschel/SPIRE (bottom row). The pixels have sizes 1 !. 0,1 !" 0,2 !. $0,6^{\prime \prime}, 10^{\prime \prime}$, and $14^{\prime \prime}$, respectively, while the corresponding Herschel's resolution is $\sim 6$ times these values. The source is detected in all six channels, and clearly extended at the PACS wavelengths. The field of view of each panel is 55", with north up and east to the left.

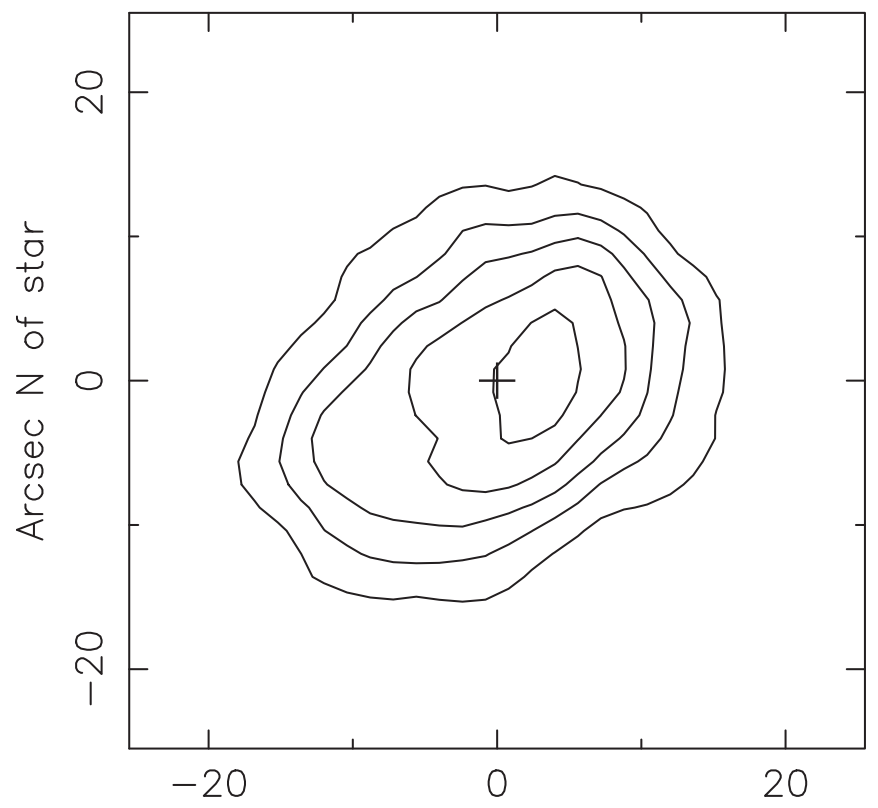

Arcsec W of star

Figure 5. Contour map of the Herschel/PACS $70 \mu \mathrm{m}$ emission from HD 202628. Contours show the $\pm 3,6,9, \ldots \sigma$ significance levels, with $\sigma=29 \mu \mathrm{Jy}$ pixel $^{-1}$.

roughly $30 \%$ brighter than at a comparable distance east of the star. Krist et al. (2012) noted the star's $\sim 0$ ".5 westward displacement from the ring center, a result confirmed by the new ALMA and HST results in this paper. Asymmetrically bright $\mu \mathrm{m}$ emission from the ring pericenter is theoretically expected on the short wavelength side of the ring's thermal emission peak (Wyatt et al. 1999; "pericenter glow"), with the effect becoming more pronounced for larger ring eccentricities. It is due to the fact that the pericenter of the ring is closer and thus hotter than the apocenter. At $70 \mu \mathrm{m}$ for a ring of eccentricity $\sim 0.1$ such as here, and depending on the dust grains properties, the pericenter is expected to be $20 \%-40 \%$ brighter than the apocenter (see Figure 2 of Pan et al. 2016).
Table 6

Photometry of the HD 202628 System

\begin{tabular}{|c|c|c|c|}
\hline $\begin{array}{l}\text { Wavelength } \\
(\mu \mathrm{m})\end{array}$ & $\begin{array}{l}\text { Flux Density } \\
\qquad(\mathrm{Jy})\end{array}$ & $\begin{array}{l}\text { Uncertainty } \\
\qquad(\mathrm{Jy})\end{array}$ & References \\
\hline 0.43 & 4.36 & & Hipparcos \\
\hline 0.55 & 7.50 & & Hipparcos \\
\hline 1.22 & 9.15 & 0.18 & 2MASS; Cutri et al. (2003) \\
\hline 1.65 & 7.78 & 0.24 & 2MASS; Cutri et al. (2003) \\
\hline 2.18 & 5.16 & 0.12 & 2MASS; Cutri et al. (2003) \\
\hline 3.6 & 2.52 & 0.17 & $\begin{array}{l}\text { WISE All-Sky catalog (Cutri } \\
\quad \text { et al. 2012) }\end{array}$ \\
\hline 11.8 & 0.23 & 0.0032 & $\begin{array}{l}\text { WISE All-Sky catalog (Cutri } \\
\quad \text { et al. 2012) }\end{array}$ \\
\hline 22.0 & 0.07 & 0.0022 & $\begin{array}{l}\text { WISE All-Sky catalog (Cutri } \\
\quad \text { et al. 2012) }\end{array}$ \\
\hline 23.8 & 0.059 & 0.0024 & $\begin{array}{l}\text { Spitzer/MIPS; Sierchio et al. } \\
\quad(2014)\end{array}$ \\
\hline 70.0 & 0.088 & 0.0044 & Herschel/PACS; this work \\
\hline 100.0 & 0.111 & 0.0055 & Herschel/PACS; this work \\
\hline 160.0 & 0.101 & 0.0051 & Herschel/PACS; this work \\
\hline 250.0 & 0.048 & 0.0019 & Herschel/SPIRE; this work \\
\hline 350.0 & 0.030 & 0.0027 & Herschel/SPIRE; this work \\
\hline 500.0 & 0.012 & 0.0010 & Herschel/SPIRE; this work \\
\hline 1300.0 & 0.000959 & 0.000096 & ALMA; this work \\
\hline
\end{tabular}

This is consistent with our findings, and makes HD 202628 only the third debris disk where this has been observed after Fomalhaut (Acke et al. 2012) and HR 4796A (Moerchen et al. 2011).

\subsubsection{Photometry and SED Modeling}

Optical and infrared photometry of the HD 202628 system is presented in Table 6. The values reported for Herschel/PACS were measured in apertures of $19 ! 2^{2}$ at 70 and $100 \mu \mathrm{m}$, and of $38.4 \operatorname{arcsec}^{2}$ at $160 \mu \mathrm{m}$, with adjacent background subtracted and uncertainties determined by the $5 \%$ accuracy of the instrument's absolute calibration. Values for Herschel/SPIRE were measured in apertures 6, 5, and 3 pixels across, background subtracted, and with uncertainties determined by the observed fluctuations in offset apertures. Extragalactic background confusion could potentially affect the flux densities, with this risk being larger at longer wavelengths.

To quantify the infrared excess of the disk it is necessary to subtract a model stellar photosphere from the infrared photometry. We used a Kurucz model with $T_{\text {eff }}=5750,[\mathrm{Fe} /$ $\mathrm{H}]=0.0$, and $\log g=4.5$ fit to the $0.43-24.0 \mu \mathrm{m}$ data points. The observed flux densities and the photospheric model fit are plotted in Figure 6. The Kurucz model fit implies a stellar photospheric emission of $19 \mu \mathrm{Jy}$ at $\lambda=1300 \mu \mathrm{m}$, about $20 \%$ less than the value observed with ALMA. This is, however, not surprising, as the few stars for which millimeter emission have been obtained all show fluxes significantly larger than what usual photospheric models predict (see, e.g., Liseau et al. 2016).

The observed spectral index of the debris disk is found to be $2.37 \pm 0.09,2.62 \pm 0.15$, and $2.64 \pm 0.20$, using the measured flux at $1.3 \mathrm{~mm}$ with ALMA, and the Herschel/SPIRE measurements at 250, 350, and $500 \mu \mathrm{m}$, respectively. On average, it is thus equal to $2.54 \pm 0.15$. Note that this is compatible with the spectral index of $2.70 \pm 0.17$ that was found for the debris disk of Fomalhaut by Ricci et al. (2012). Using their Equation (1), and adopting their values for the dust 


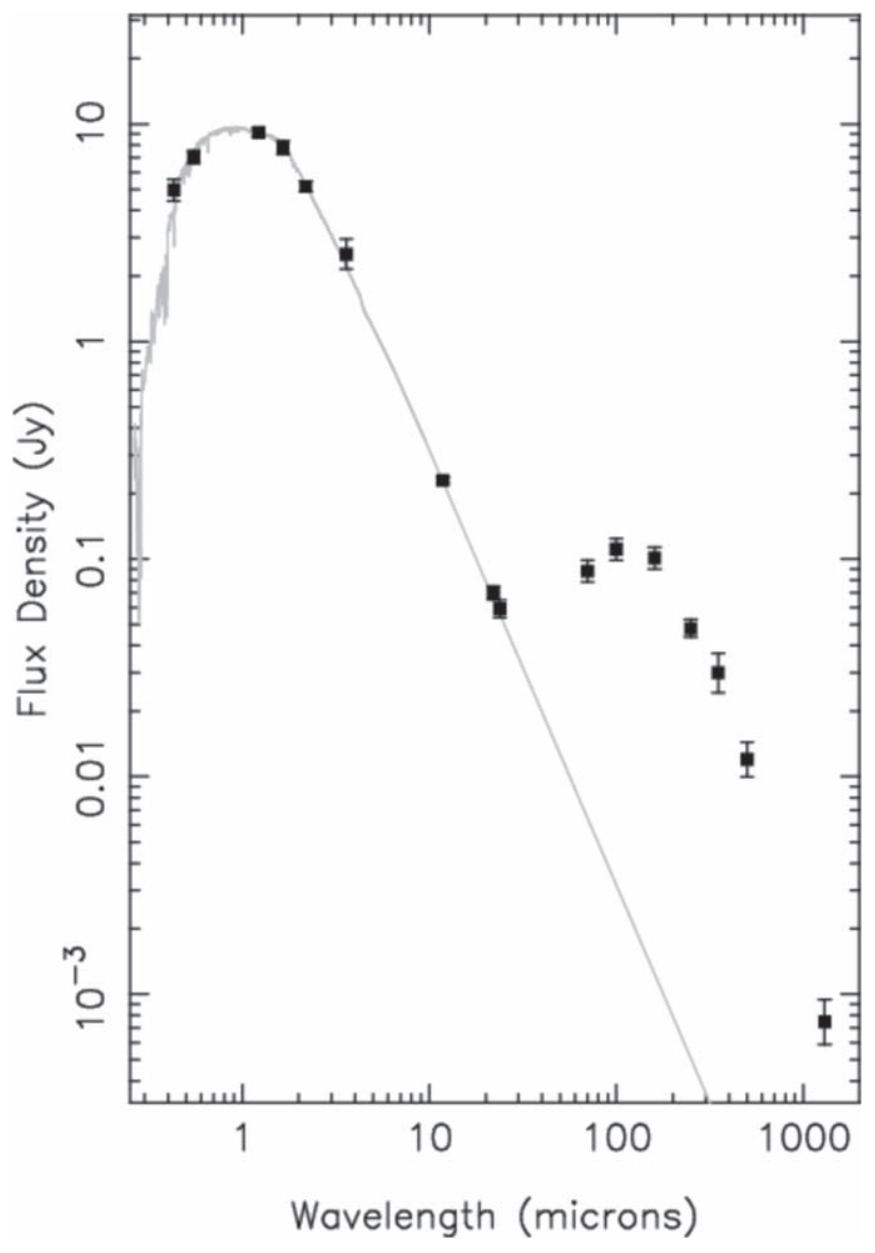

Figure 6. Photospheric model fit and observed flux densities.

opacity and Planck function spectral indices $\left(\beta_{s}=1.8 \pm 0.2\right.$ and $\alpha_{\mathrm{Pl}}=1.84 \pm 0.02$ ), we find that the index $-q$ of the grain size distribution power-law in the debris disk of HD 202628 is $-3.39 \pm 0.15$, which is in accordance not only with the theoretical Dohnanyi value of -3.5 for a steady state collisional cascade (Dohnanyi 1969), but also with the average value actually observed for debris disks $(\langle q\rangle=3.36 \pm 0.02$, MacGregor et al. 2016). On the other hand, it is not compatible with the scenario proposed by Kenyon \& Bromley (2015), in which a Super-Earth is currently forming at large distance from its host star in this system, as this would require a much steeper grain size distribution, with a power-law index of $q \sim 4.5-5.5$.

The infrared SED can be modeled to further constrain the properties of the circumstellar dust. Following the approach detailed in Krist et al. (2010), and using the ring size and structure information provided by the HST images (see Section 3.3.2), a three-zone emission model was constructed with three parameters to describe the dust size distribution: $a_{\min }, a_{\max }$, and the dust size distribution power-law spectral index $\gamma . a_{\min }$ is a free parameter set to the same value in all three zones, while $\gamma$ was fixed to the theoretical Dohnanyi value of -3.5 . While the $a_{\text {min }}$ parameter has an important effect on the SED, $a_{\max }$ only affects the total millimeter flux, and to a lesser extent, the slope of the SED at the Herschel/SPIRE and ALMA wavelengths. Therefore, the most sensitive parameter, $a_{\text {min }}$, was iterated first until a reasonably close SED was found, and $a_{\max }$ was subsequently set on a second phase. In the central zone corresponding to the parent body ring seen by ALMA,

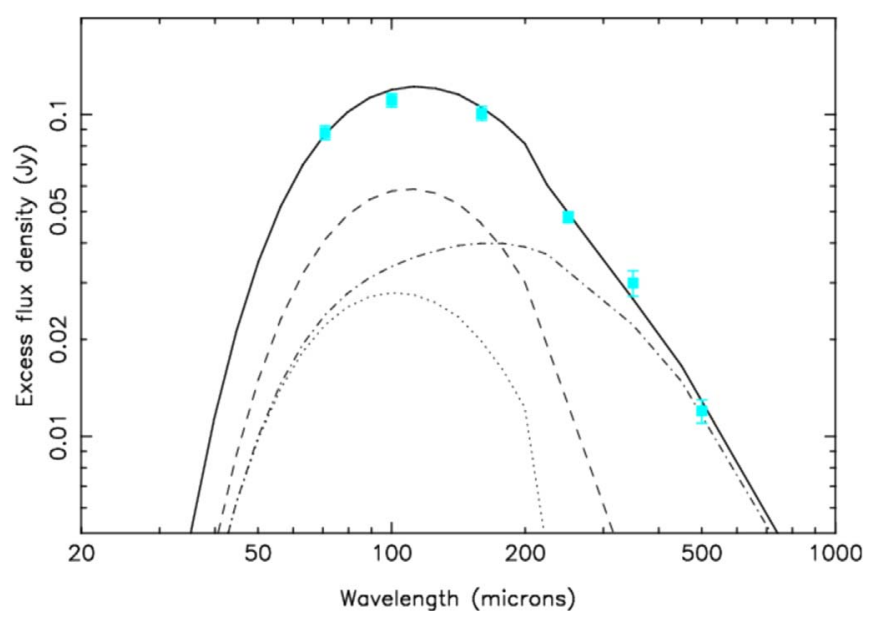

Figure 7. Spectral energy distribution of the infrared excess emission from the HD 202628 debris ring (points) and the best-fit emission model constrained by the three zones derived from the distribution of scattered light. The contributions of the inner, middle, and outer zones are shown by the dotted, dotted-dashed, and dashed lines, while the total model emission is shown as a solid line.

$a_{\max }$ was set to $1 \mathrm{~mm}$. For the inner and outer zones, $a_{\max }$ was arbitrarily set to $32 \mu \mathrm{m}$, that is, 10 times the minimum grain size, based on the assumption that radiation pressure and Poynting-Robertson (PR) drag cannot effectively transport larger grains away from their formation region in the central zone. Under these assumptions, and using Mie spheres with astronomical silicate composition, the resulting model fit is shown in Figure 7 along with the excess emission values obtained after subtracting the stellar photospheric model from the flux densities in Table 6. $a_{\min }$ is found to be $3.2 \mu \mathrm{m}$. As seen in other systems, this value is a few times larger than the theoretical blowout size for a star with $1 L_{\odot}$ (Booth et al. 2013; Pawellek et al. 2014; Pawellek \& Krivov 2015). The fractional IR luminosity of the best-fit model is $7 \times 10^{-5}$, with just over half of this coming from the inner and middle zones.

\subsection{New HST/STIS Observations}

The images in Figure 8 demonstrate the improvement gained with the additional $7.7 \mathrm{hr}$ of integration time in 2014 and 2015 compared to the original $1.3 \mathrm{hr}$ image from 2011. The inner edge of the ring is better defined and the disk can be traced out further from the star. In the disk ansae the mean $\mathrm{S} / \mathrm{N}$ is $\sim 10$, and the mean surface brightness there is $\sim 65$ times less than the stellar PSF's. All of the images show streaks radiating from the star that are subtraction residuals due to PSF mismatches caused by pointing errors and optical aberration variations over time. The disk is likely smooth. Anything interior to the ring may be artifacts as well-there is no infrared excess to suggest interior material that would be visible in these images. Along the ring minor axis the disk suffers the most from subtraction artifacts, and the fainter SW side is especially noisy. A brightening on the NE side of the ring along the minor axis is very likely an artifact. This region is the closest in apparent separation to the star and thus is the most likely to be subject to PSF subtraction artifacts. Indeed, and unsurprisingly, such brightenings appear around this region in the 2014 and 2015 data (which is masked by a spider in 2011), though never at the same position between years or between months within the same year, which is consistent with it being a subtraction 

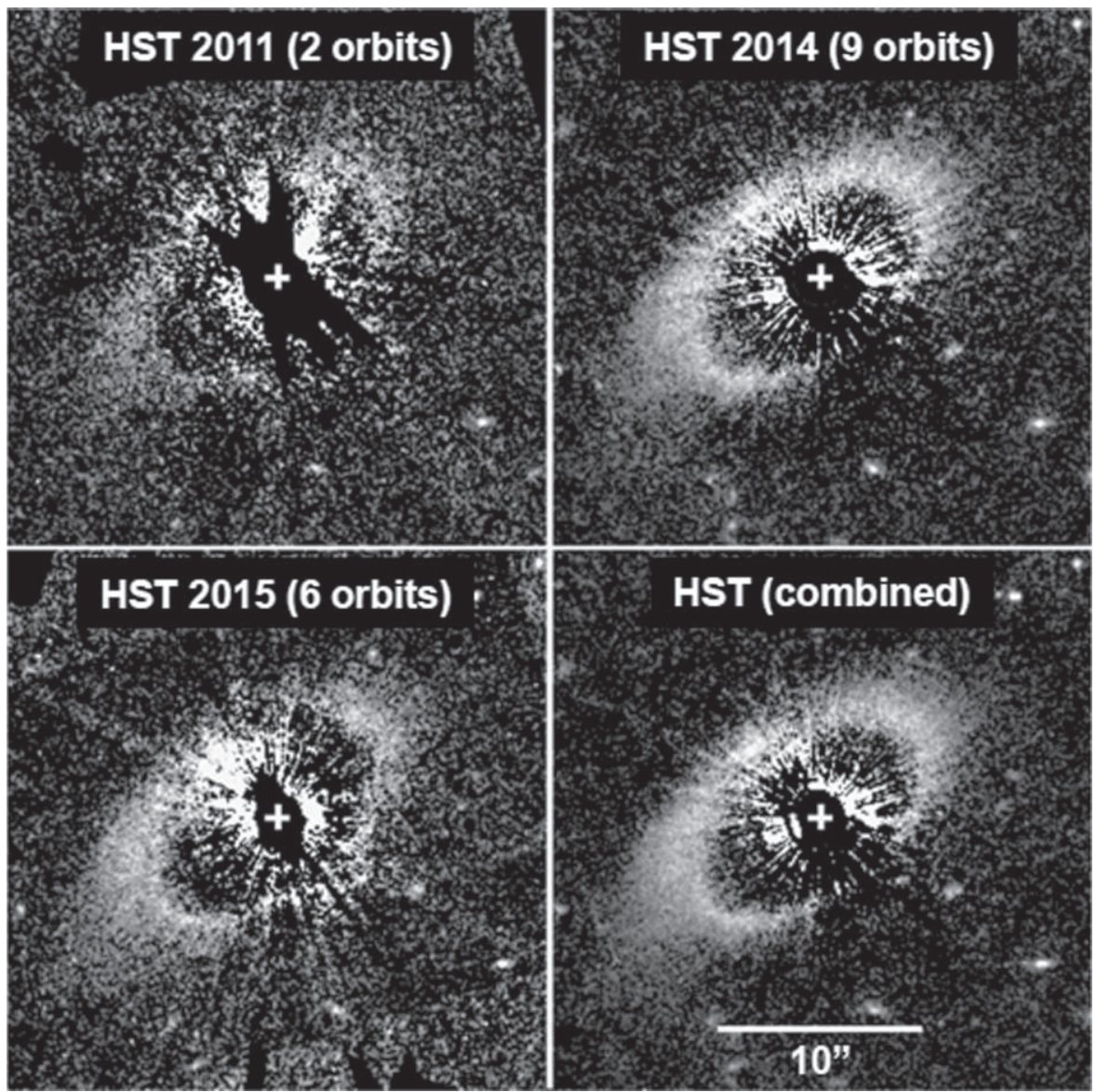

Figure 8. HST STIS coronagraph images of the HD 202628 disk derived from ADI post-processing of data from each epoch. In the lower right is the combination. The square-root of the intensity is shown. North is up, and a cross marks the position of the star.

artifact. A calibrated surface brightness map is shown in Figure 9.

There are numerous background galaxies and some point sources visible in the full $50^{\prime \prime}$-wide $H S T$ image, but there is no correspondence between any of them and sources seen in the ALMA image.

\subsubsection{Deprojection}

Deprojection of the scattered-light emission and accurate knowledge of the geometry of the disk as provided by ALMA can teach us further about the dust grains distribution and properties. Indeed, if the debris disk was a circular ring with a uniform azimuthal density distribution, the observed deprojected scattering pattern would be left-right symmetrical about the line of sight, with the near side of the ring to the observer being brighter than the far side if there is forward scattering. If the ring is not circular but eccentric as is the case here, the side closer to the star will be brighter if the scattering is isotropic (pericenter glow). These effects are illustrated in Figure 10 (top left panel), where we display a model of eccentric ring seen face-on, with uniform azimuthal density distribution and moderate forward scattering. The scattering pattern is not exactly symmetrical about the line of sight, but instead is symmetrical about a line that is rotated toward the periastron. Note that as the degree of forward scattering increases, the brightest part of the ring will rotate from periastron toward the line of sight.
Using the inclination $(i)$ and position angle of the ascending node $(\Omega)$ derived from the ALMA image, the ALMA and HST images were deprojected via interpolation to a face-on orientation, as shown in Figure 10 (top right and bottom left panels, respectively). We produced as well an additional map where each pixel in the deprojected $H S T$ scattered light image was multiplied by the square of its physical distance from the star (as derived from the disk geometry seen with ALMA), compensating for the stellar illumination falloff, and shown in Figure 10 (bottom right panel). Assuming an optically and -vertically thin disk, the result is a map of the relative scatterer density modulated by the scattering phase function of the dust grains. In other words, this map is freed from the effects of stellar illumination, that is, of the pericenter-glow, and provides a better representation of the disk actual density, though not entirely freed from the effects of the scattering properties of the grains. As compared with what would be expected from the disk shown in the model image, the deprojected HST image shows that the bulk of the light distribution is instead skewed away from the star and to the left of the line of sight, forming an arc extending radially outward from north to east (counterclockwise), and which can be explained most easily by a higher relative dust density there (assuming azimuthally uniform grain properties). This feature is very similar to the one observed in the debris disk of HD 181327, which geometry is precisely the one expected from the distribution of small grains released from a high-mass collisional event (Kral et al. 2013; Jackson et al. 2014). This led Stark et al. (2014) to attributed this feature 


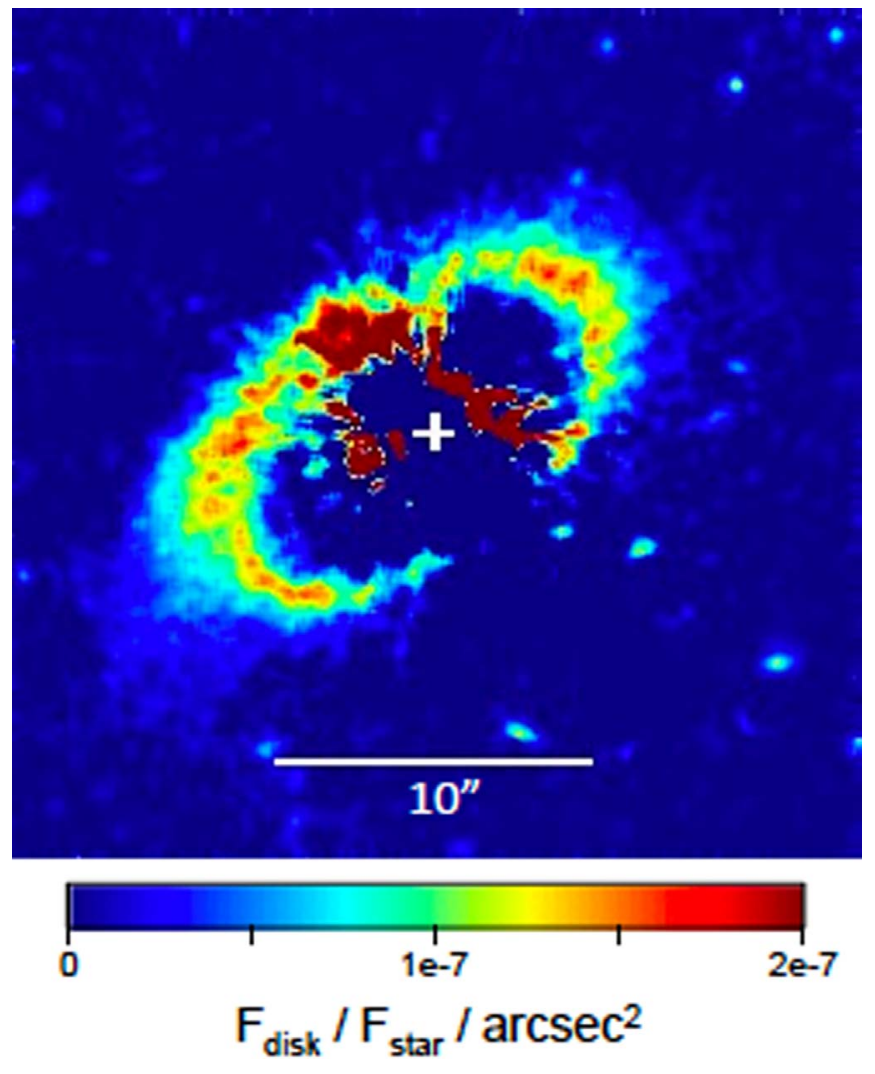

Figure 9. HST STIS coronagraph image of the HD 202628 disk in units of disk surface brightness relative to the total stellar flux. North is up, and a cross marks the position of the star.

to a massive collision within the debris disk of HD 181327, and hence could be the case for that of HD 202628 as well.

While we will focus on the effects of the scattering-phase function in the next section, we can further study the disk azimuthal density distribution using radial profiles. Figure 11 shows the surface brightness profile derived by computing at each radius from the star the azimuthal median values in $36^{\circ}$ sectors oriented along the horizontal axis of the deprojected $H S T$ image. Recall that this is not the direction of the intrinsic major axis of the ring but rather the axis of the ascending nodes. We precisely chose this direction because the scattering angle along this axis is the same $\left(90^{\circ}\right)$, and thus the modulation from the scattering phase function is identical on both sides and has no impact on the relative brightness of each side in the plot. This leaves us with a profile that can be impacted by both the disk eccentricity and its azimuthal density distribution, and freed from the scattering-phase function effects. Since the ring is indeed eccentric, its periastron is expected to be more illuminated and thus brighter in scattered light than the apastron. This effect is shown in Figure 11 (solid line), and obtained assuming a ring model that is azimuthally uniform. One can see that the observations (dashed line) show instead that the NW and SE sides have comparable brightnesses. This tells us that the ring is in fact not uniform in azimuth, and that its apocenter exhibits an overdensity, as already hinted by the deprojections shown in Figure 10.

We can take this reasoning a step further by using a similar plot and the deprojected $H S T$ relative density map, that is, the map which is freed from the pericenter-glow effects. This plot is displayed in Figure 12 and shows an enhanced density on the SE side, that is, at apastron: this plot shows that the overdensity translates into a ring actually extending further out there, as is expected from eccentric debris disks seen in scattered light, and due to radiation pressure (Lee \& Chiang 2016). First, small dust grains such as those seen in scattered light are subject to radiation pressure, and are on more eccentric orbits than their parent bodies. Therefore, their distribution extends further out radially than that of the parent grains seen with ALMA. second, an extended apastron in scattered light is due to the fact that the small dust grains are preferentially released on orbits that are apsidally aligned, that is, they are being released in majority at their parent bodies periastron (Lee \& Chiang 2016).

\subsubsection{Scattered Light Models}

To qualitatively evaluate various ring properties, such as the scattering phase function and the effect of pericenter glow, we constructed a scattered light model using the measured ring properties. These models were also useful to evaluate the impact of self-subtraction during post-processing, as discussed in Appendix B. We emphasize the qualitative nature of our model-to-data comparisons presented here: more involved modeling, which would include simultaneously fitting both the HST and ALMA data and would account for the apparent azimuthal distribution asymmetry, is left for future studies. A simple single-scattering, three-dimensional model appropriate for optically thin disks was used with a constant Gaussian vertical distribution with a full-width-half-maximum of $2 \mathrm{au}$ (the disk is assumed to be so geometrically vertically thin relative to its radial extent that this assumption is largely unimportant in regards to the morphology of the simulated image). Based on the shape of the NW density map profile, the model was composed of three contiguous annular zones, each defined by its inner semimajor axis $(a)$ and a radial density power law: (1) $132 \mathrm{au} \leqslant a \leqslant 157 \mathrm{au}, \quad r^{+9.0}$; $\quad$ (2) $157 \mathrm{au} \leqslant a \leqslant 172 \mathrm{au}, r^{-2.5} ;$ (3) $172 \mathrm{au} \leqslant a \leqslant 268 \mathrm{au}, r^{-4.5}$. The dust was distributed using the measured eccentricity parameters.

Various scattering phase functions were used: the simple Henyey-Greenstein (H-G) function (Henyey \& Greenstein 1941), the Hong (1985) function for zodiacal dust (their $\nu=1$ case), and the Hedman \& Stark (2015) function for Saturn's G ring. The Hong and G-ring functions are each the sum of three H-G functions with various choices of forward scattering parameters $(g)$ and corresponding weights, and they produce similar results, though the Hong function has slightly more backscatter. See Hughes et al. (2018) for a discussion of the limitations of the single $\mathrm{H}-\mathrm{G}$ representation for debris disks.

Figure 13 shows the HST data at two different intensity stretches along with the similarly displayed models. Note that in the isotropic scattering $(g=0)$ case, the ring is brightest to the $\mathrm{W}$, since that is the side closest to the star. Once moderate $(g>0.2)$ forward scattering is introduced, the brightest portion moves toward the line of sight. The Hong and G-ring phase functions clearly have significant forward scattering (their primary $\mathrm{H}-\mathrm{G}$ terms have $g=0.995$ and 0.7 , respectively). One must avoid mistaking the bright spot along the NE edge of the data as an indicator of strong forward scattering. It is not located along the line of sight to the star, and it is $4 . .7$ from the star while in the Hong and G-ring models the brightest spots are at 3".9. This further indicates that this feature is likely a PSF subtraction artifact. Even if it were real, it appears more concentrated than the spots in the models. Ignoring that spot, the ring appears much more azimuthally uniform in brightness 


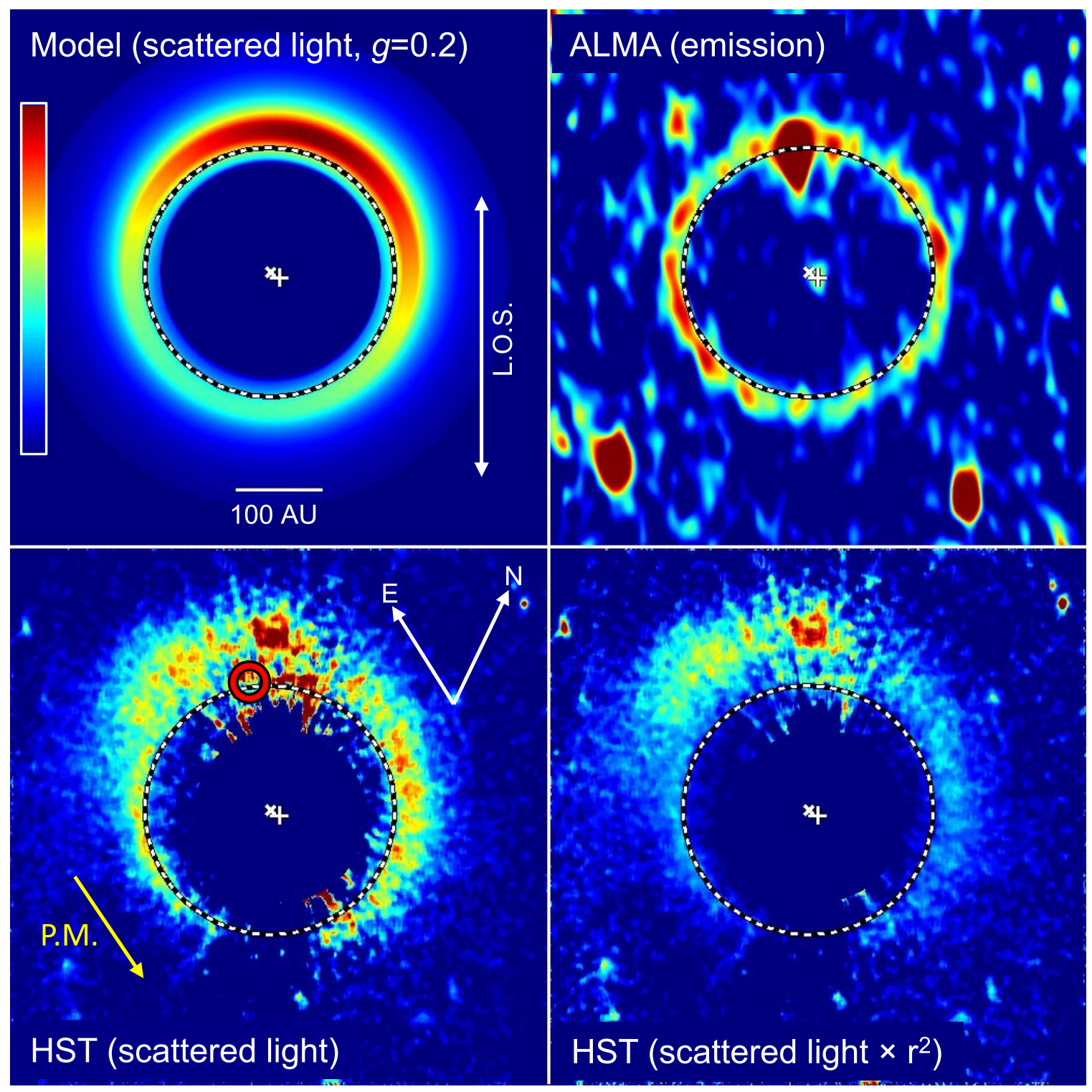

Figure 10. HST (PSF-subtracted) and ALMA images of the HD 202628 disk deprojected to a face-on orientation. The horizontal axis is along the line of ascending nodes. The line of sight (L.O.S.) is along the vertical axis. The position of the star is marked by a cross + and the geometric center of the ring by an $\times$. The fitted ellipse to the inner edge of the ALMA ring is represented by a dashed line in each panel. All of the images are displayed with an arbitrary linear intensity scale. (Top left) Deprojected scattered light model with moderate forward scattering $(g=0.2)$, assuming that the top side of the ring is closest to the observer. (Top right) ALMA emission image. (Bottom left) Deprojected HST scattered light image. The red circle marks where the suspect point source in the ALMA image would be in 2014 due to proper motion of the star if it were a background object, while the arrow represents the deprojected apparent star's proper motion (assuming no out-of-sky motion). (Bottom right) The deprojected HST image multiplied by the square of the distance of each pixel from the star, compensating for stellar illumination falloff and representing the relative density distribution of scatters modulated by the scattering phase function.

than in the Hong or G-ring models, suggesting a more isotropic scattering function over the range of observable scattering angles $(g<0.2)$, as suggested by Krist et al. (2012) and Schneider et al. (2016). However, the strong intensity stretch images better match with the more forward-scattering functions, most notably along the presumably far side (SW) of the ring. In the data this side appears truncated when compared to any of the models. The dust distribution is clearly asymmetric, as evidenced by the extension to the SE, which, as mentioned in the previous section, is well reproduced in synthetic images of scattered light emission of eccentric debris disks (Lee \& Chiang 2016).

\section{Constraints on the Distant Eccentric Perturber}

In light of the new constraints for this system, that is, the fact that the ring's eccentricity is found smaller than in previous observations ( $e=0.18$ Krist et al. 2012), the accurate radial location of its inner edge, as well as the fact that Gaia has given new constraints on the star's distance, ${ }^{16}$ we will base our analysis on the previous theoretical modeling work by Pearce \& Wyatt (2014) and update the constraints that can thus be set on the eccentric belt-shaping perturber (mass $m_{\mathrm{p}}$, semimajor axis $a_{\mathrm{p}}$, and eccentricity $e_{\mathrm{p}}$ ).

\footnotetext{
${ }^{16}$ Note that the new Gaia distance value of $23.8 \mathrm{pc}$ is only $2.5 \%$ smaller than the previous Hipparcos value of $24.4 \mathrm{pc}$, and therefore, will only have little impact on the constraints we derive as compared to the accurate knowledge of the ring's geometry.
} 


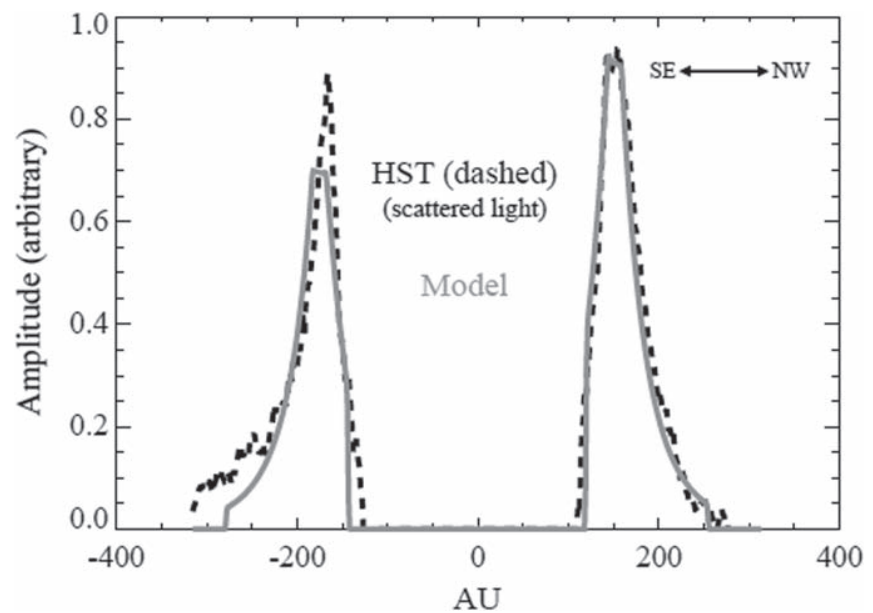

Figure 11. Plots of the azimuthal median intensity vs. radius from the star measured in $36^{\circ}$ sectors aligned along the horizontal axis (line of nodes) of the deprojected HST STIS coronagraph image of the HD 202628 disk (dashed line) and a corresponding deprojected scattered light model (solid gray line). The intensity scale is arbitrary, and the two plots have been normalized to match on the NW side. The model, which is azimuthally uniform, is brighter to the NW because that is the side closer to the star (the degree of forward scattering is irrelevant since both sides are at the same scattering angle).

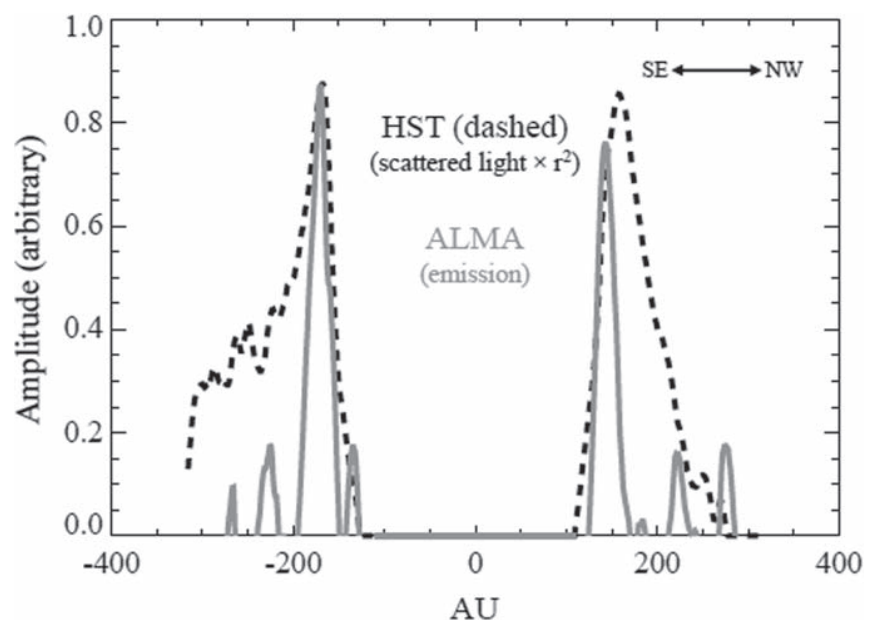

Figure 12. Plots of the azimuthal median value vs. radius from the star measured in $36^{\circ}$ sectors aligned along the horizontal axis (line of nodes) of the deprojected HD 202628 HST relative density map (dashed line) and the deprojected ALMA image. The vertical scale is arbitrary, and the two plots have been normalized to match on the SE side.

We will discuss as well the nature of the source S1, which, with its position just inside the ring, that is, on the expected path of an eccentric perturber, and along with other arguments we develop hereafter, gives us good reason to think it might actually be circumplanetary material surrounding the perturber. We will show how this would allow us to alleviate degeneracies and fully characterize the mass and orbital properties of the belt-shaping perturber.

\subsection{Theoretical Constraints}

Setting precise constraints on the eccentric ring perturber inferred around HD 202628 requires detailed investigations involving complementary theoretical and numerical dynamical modeling, along with the production of synthetic images for comparison with our observations. This type of work has been carried out by Pearce \& Wyatt (2014) and Thilliez \& Maddison
(2016), respectively. However, these studies were based on the geometry of the inner edge as given by HST in 2012, which was found to be more eccentric than our ALMA and deeper HST observations show, and for which the constraints on the radial distance of the inner edge of the star were not as precise as our ALMA observations reveal. Moreover, these studies relied on the distance of the star as found by Hipparcos (van Leeuwen 2007), whereas Gaia has given new constraints on the distance of the system that slightly modify the distance of the inner edge of the disk in astronomical unit, and therefore, the constraints derived on the perturber's semimajor axis. Finally, these constraints were derived considering an age of 2.3 Gyr for the system, whereas new estimates give an age of 1.1 Gyr (see Appendix A). While extensive parametric exploration and $\mathrm{N}$-body simulations are beyond the scope of the present paper, we nevertheless adopt the theoretical framework of Pearce \& Wyatt (2014), which we summarize hereafter, in order to update the theoretical constraints that can be set on the eccentric belt-shaping perturber at play in the system of HD 202628.

Constraints can be set on the perturber's mass $m_{\mathrm{p}}$, semimajor axis $a_{\mathrm{p}}$, and eccentricity $e_{\mathrm{p}}$, relying on secular LaplaceLagrange theory of perturbations, and considering the following:

The perturber forces the eccentricity of the debris ring-The eccentricity $e_{\mathrm{f}}$ forced onto constituents of the debris ring of semimajor axis $a$ reads:

$$
e_{\mathrm{f}} \simeq \frac{5}{4} \alpha e_{\mathrm{p}}
$$

(Pearce \& Wyatt 2014, Equation (1) of), where $\alpha=a_{\mathrm{p}} / a$. From Equation (1), and considering that the inner edge of the ring, with semimajor axis $a=143.1$ au, has an eccentricity forced to $e_{\mathrm{f}}=0.09$, one can then derive sets of planetary semimajor axis and eccentricity $\left(a_{\mathrm{p}}, \mathrm{e}_{\mathrm{p}}\right)$ that will induce the observed eccentricity at the disk inner edge, as shown in the right panel of Figure 14.

The perturber shapes the ring inner edge-The zone around a planet where mean-motion resonances overlap is called the chaotic zone. Small bodies in this region suffer close encounters, which results in a clearing of this zone. When modeling the shaping of a debris ring inner edge by a perturbing planet, this inner edge lies at the outer boundary of the planet's chaotic zone. The width of a chaotic zone depends primarily on the mass and semimajor axis of the planet which is responsible for it, and therefore, one can derive constraints on these two parameters as a function of the inner edge semimajor axis. There are different formulae that can be used to derive these constraints, based on previous works, however, as pointed out by Pearce \& Wyatt (2014), most of these results hold for low planet eccentricities, and therefore, they established another criterion which we will use here. This criterion, which was validated numerically, is that the apastron $Q_{\text {edge }}$ of the disk inner edge should lie at approximately five times the Hill radius of the planet at apastron $R_{\mathrm{H}, \mathrm{Q}}$ from the planet's apastron $Q_{\mathrm{p}}$, which reads :

$$
Q_{\text {edge }} \approx Q_{\mathrm{p}}+5 R_{\mathrm{H}, \mathrm{Q}}
$$



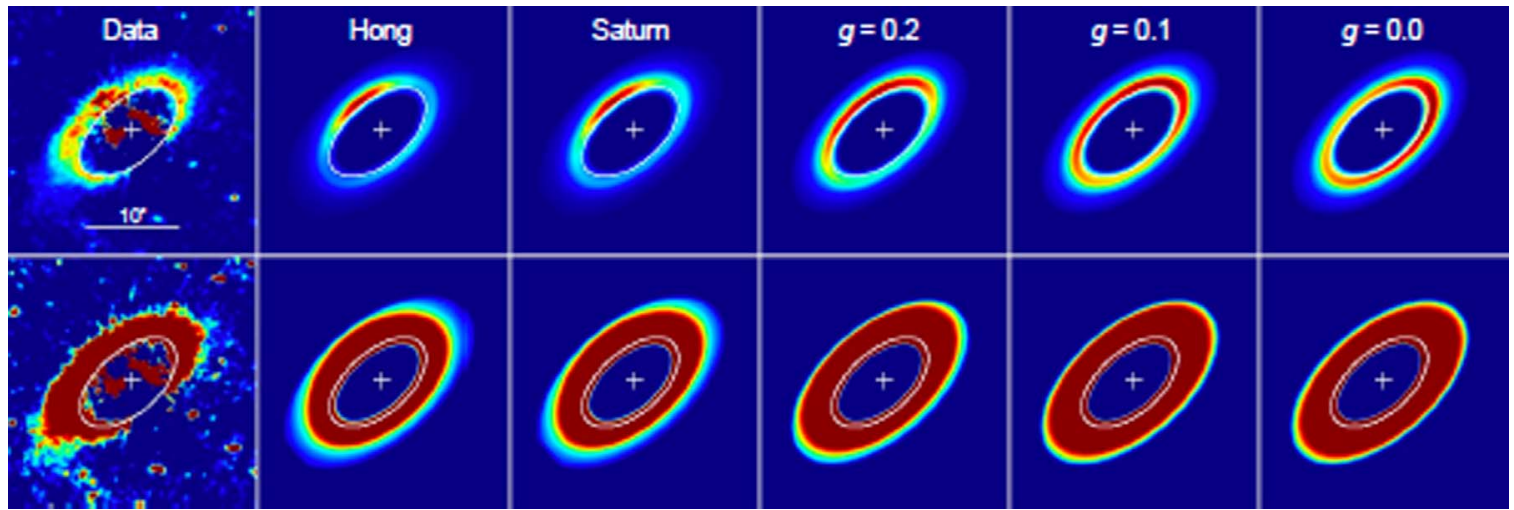

Figure 13. Comparison of the HST image of HD 202628 and scattered light models with different scattering phase functions. The top row shows the images with scaling between the minimum and approximately maximum value of each. The bottom row images are truncated at a much lower maximum value to emphasize fainter signal and the isophote of the brightest portions of the disk. The phase functions are Hong zodiacal light, G-ring, and Henyey-Greenstein $(g=0.2 ; 0.1 ; 0.0)$.

where $R_{\mathrm{H}, \mathrm{Q}}$ is defined by :

$$
R_{\mathrm{H}, \mathrm{Q}} \approx a_{\mathrm{p}}\left(1+e_{\mathrm{p}}\right)\left[\frac{M_{\mathrm{p}}}{\left(3-e_{\mathrm{p}}\right) M_{\star}}\right]^{\frac{1}{3}} .
$$

For more details, see Equations (9) and (10), as well as Appendix B of Pearce \& Wyatt (2014). As displayed on the left panel of Figure 14, one can derive an additional constraint on the mass $m_{\mathrm{p}}$ the planet should have to create the disk inner edge when having a semimajor axis and eccentricity compatible with forcing the disk inner edge eccentricity to the observed value, by combining Equations (2) and (3) with Equation (1).

The perturber has acted long enough upon the debris disk for the eccentric ring to be fully formed and well definedWhen an eccentric perturber starts acting upon a debris disk (which is assumed to be composed of planetesimals that are initially on low eccentricity, unaligned orbits), the appearance of a well defined eccentric ring is preceded by the appearance and disappearance of spirals. As this phenomenon results from the orbital precession of the constituents of the debris disk, the characteristic timescale associated with this phenomenon is the secular precession timescale, which reads:

$$
t_{\mathrm{sec}} \approx 4 T_{\mathrm{p}}\left(\frac{m_{\mathrm{p}}}{M_{\star}}\right)^{-1} \alpha^{-5 / 2}\left[b_{3 / 2}^{(1)}(\alpha)\right]^{-1},
$$

where $T_{\mathrm{p}}$ is the perturber's orbital period, $M_{\star}$ is the mass of the central star, and $b_{3 / 2}^{(1)}$ is a Laplace coefficient (see Equation (17) of Pearce \& Wyatt 2014).

The perturber has had the time to clear its surroundings for the inner edge to be sharpened-Therefore, we consider the diffusion timescale, which is the timescale necessary for a planet to form the inner edge by ejecting material in its surroundings. As derived by Pearce \& Wyatt (2014), this timescale reads (see Equation (18) of Pearce \& Wyatt 2014).:

$$
t_{\text {diff }} \sim 0.01 T_{\mathrm{p}} \alpha^{\frac{1}{2}}\left(\frac{M_{\mathrm{p}}}{M_{\star}}\right)^{-2} .
$$

In their numerical simulations, Pearce \& Wyatt (2014) noted that it took ten times the higher timescale between the secular timescale and the diffusion timescale to form an inner edge and give its eccentricity to the disk. As shown in the right panel of Figure 14, one can set constraints on the minimum mass of the belt-shaping perturber, as below this mass, it would take timescales larger than the age of the system for the planet to shape the edge of the debris disk and spirals to disappear.

Note that we chose here to focus on the most usual assumption of a single inner perturber, as a thorough dynamical modeling is beyond the scope of this paper. Other possibilities are that the debris disk is shaped by an outer eccentric perturber (Faramaz et al. 2014), or, because of its sharp outer edge, and similar to Fomalhaut, by a pair of shepherding planets (Boley et al. 2012). In addition, the eccentricity derived for the perturber should be considered to be a minimum and possibly larger. Indeed, it was shown in Faramaz et al. (2014) that a debris disk eccentricity tends to relax to smaller (yet non-zero) values than the predicted forced eccentricity when acted upon by an eccentric planet on timescales of several $100 \mathrm{Myr}$ and up to $1 \mathrm{Gyr}$.

\subsection{Could S1 be Circumplanetary Material Surrounding the Belt Perturber?}

While the ALMA field contains several sources that are all marginally resolved, the brightest one, S1, caught our attention. Indeed, its location is consistent with the expected orbit of the belt-shaping perturber, just interior to the circumstellar ring; therefore, if bound to the star, it could be explained by the existence of circumplanetary ring system surrounding the beltshaping perturber.

No obvious point source is detected with $H S T$ that is consistent with S1. This is unsurprising because if S1 was part of the system, it could not have been seen in these observations, as its offset of $\sim 3.15$ from the star in the NE direction corresponds to a region which suffers from stellar artefacts and is partially masked by the $H S T /$ STIS coronograph. On the other hand, as the star exhibits a high proper motion (242.190 $\mathrm{mas} \mathrm{yr}^{-1}$ in R.A. and $21.63 \mathrm{mas} \mathrm{yr}^{-1}$ in decl.) (Gaia Collaboration et al. 2018), a background object would have been offset of $\sim 4$ ". $5 \mathrm{NE}$ of the star back in 2011 May on HST observations, which show no sign of associated visible light emission at this location, thus placing an upper limit of $V>25$ on a background source at this location. This cannot completely rule out the possibility of the source being a background galaxy, however, as background galaxies seen at submillimeter wavelengths do not obviously show an associated visible light emission.

According to millimeter-counts in Band 6 and using the Schechter function (Carniani et al. 2015), we would expect 2.4 background sources brighter than S3 (the faintest of the 

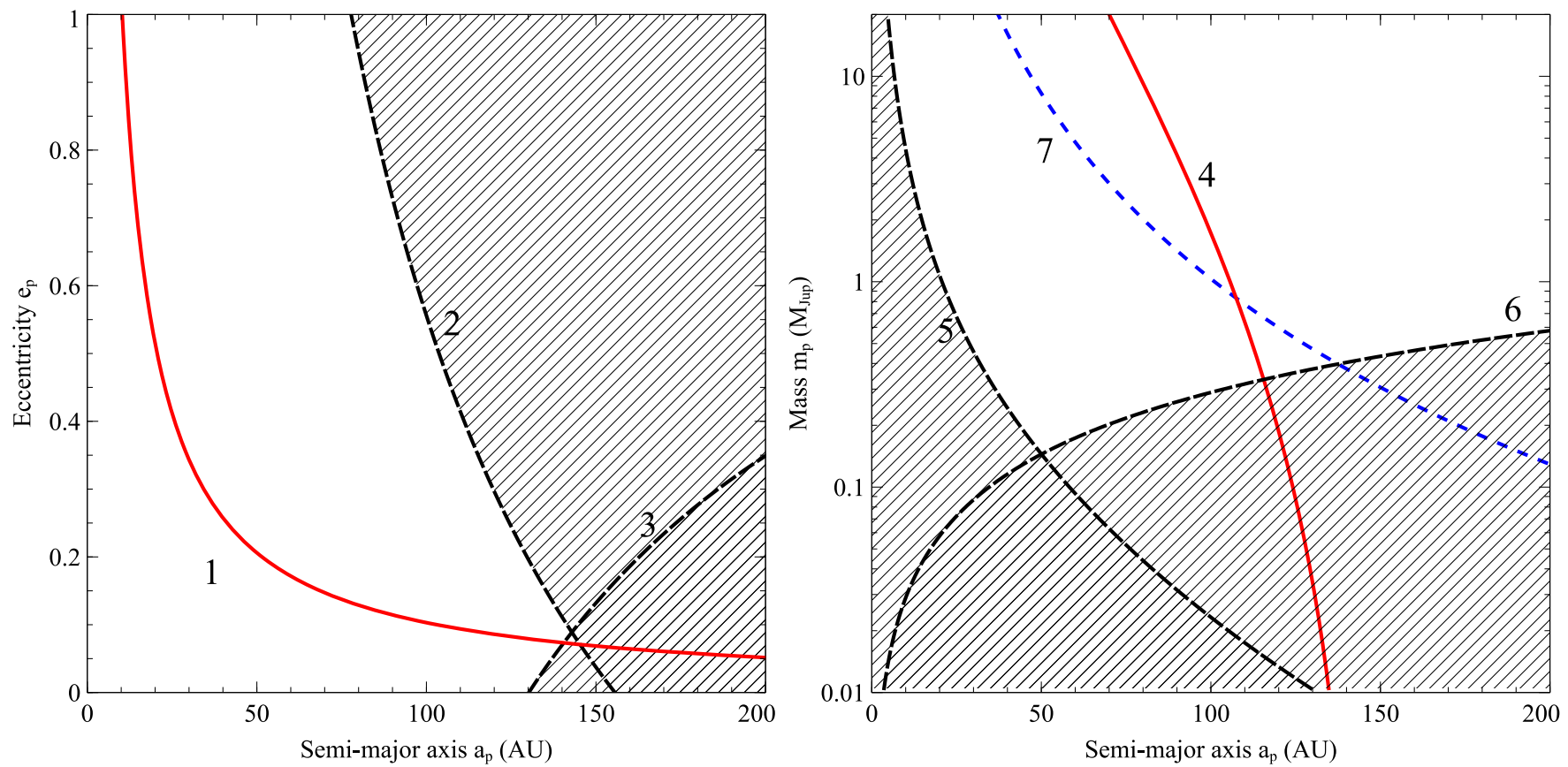

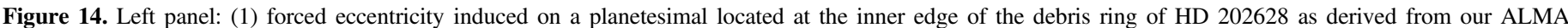

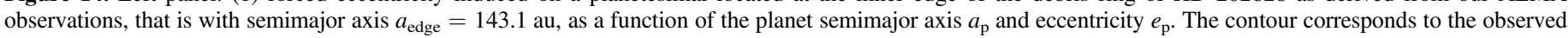

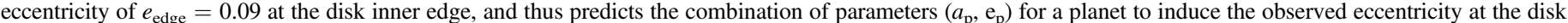

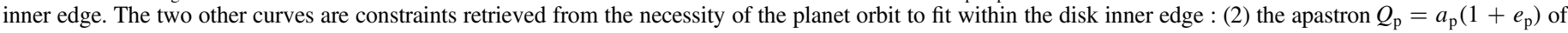

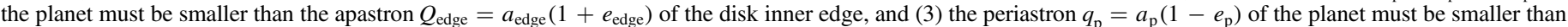

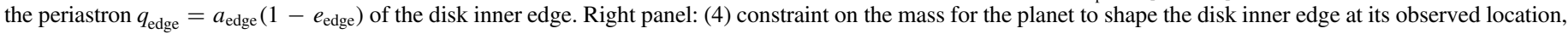

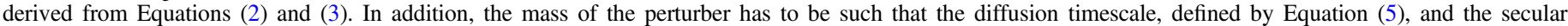

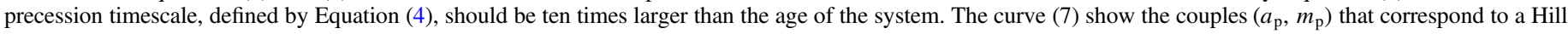
radius compatible with the half extent of the source S1 (see Equation (6) and Section 4.2).

sources) within the ACA Half Power Beam Width (HPBW) of diameter 41 ". 4 in Band 6 (the region delimited by the gray line on the images, that shows the 50\% sensitivity level). Therefore, instead of our circumplanetary ring hypothesis, the presence of a total of four sources in our observations may instead mean that HD 202628 is aligned with galaxies that are part of a cluster, which is the case for $10 \%-20 \%$ of galaxies (Tempel et al. 2012).

This source is not detected in Herschel/PACS resolved observations at 70 and $100 \mu \mathrm{m}$, which means that if it were circumplanetary material, its smallest dust grains would be larger than $\sim 100 \mu \mathrm{m}$. Note that this would be in accordance with what we know about circumplanetary rings in the solar system, as the smallest particles in Saturn's A ring have been found to be millimeter sized, possibly down to $500 \mu \mathrm{m}$ accounting for error bars (Becker et al. 2016). It may nevertheless possess emission at Herschel/SPIRE wavelengths, for which no resolved image has been obtained, but only photometric measurements, which might actually explain the discrepancy between the flux we predicted for the debris ring and the one we observed. Indeed, we based our Band 6 continuum flux density estimates for the debris disk on these Herschel photometric measurements. Following the modeling strategy used for the debris disks of HD 207129 (Krist et al. 2010) and HD 92945 (Golimowski et al. 2011), the HST image was used to define the spatial distribution of the dust and the dust grain properties were adjusted until the model was consistent with the far-infrared SED. Extrapolating the Herschel/SPIRE 250-500 $\mu \mathrm{m}$ spectral index to $1.3 \mathrm{~mm}$, which was found to be $2.0 \pm 0.5$, as expected from a solid population in collisional cascade, we had estimated a total $1.3 \mathrm{~mm}$ continuum flux density of 1.1-2.6 mJy. We therefore expected to observe the circumstellar ring with a $\mathrm{S} / \mathrm{N}$ of $\sim 5$ if our predicted flux was distributed in the same way as $H S T$ scattered light, and expected this $\mathrm{S} / \mathrm{N}$ to be 10 or higher if the circumstellar ring was much narrower at millimeter wavelengths, similarly to what our ALMA Cycle 4 observations reveal. However, we find instead that the circumstellar ring is mostly detected with a $\mathrm{S} / \mathrm{N}$ of $\sim 3-4$, and that the disk total flux integrated within the $2 \sigma$ contours is found to be $959 \pm 96 \mu \mathrm{Jy}$. While this is still formally compatible with our estimates, it nevertheless lies at their low end. If the compact source possessed emission at SPIRE wavelengths, we extrapolated the circumstellar debris ring flux from overestimates at these wavelengths, and subsequently would have overestimated the circumstellar disk flux in Band 6. Note that with an integrated flux of $285 \pm 18 \mu \mathrm{Jy}$ for $\mathrm{S} 1$, the total flux disk+S1 is compatible with the flux we had predicted for the debris disk.

Assuming a temperature of $30 \mathrm{~K}$ and a dust opacity of $2 \times 10^{-4} \mathrm{~cm}^{2} \mathrm{~g}^{-1}$, the source's measured flux density would correspond to a circumplanetary ring system containing a minimum ${ }^{17}$ mass of $10^{-8} M_{\odot}$, that is, 1000 times the mass of Saturn's rings, or about one-third of the Earth's Moon. This would not be the first time such a massive circumplanetary ring system has been detected, as a massive (0.4-8, and possibly up to 100 Moon's masses) ring system has been inferred in the 2MASS J14074792-3945427 system through a complex

\footnotetext{
17 This mass is that in grains of size comparable to the wavelength of the observations $(\sim 1 \mathrm{~mm})$, and hence does not encompass all the grain size range expected in a disk.
} 
series of eclipses of the host star (Mamajek et al. 2012; Kenworthy \& Mamajek 2015).

Whether the source S1 is co-moving or not cannot be ascertained without second epoch observations. However, if it were to be linked to the system, it would permit us to alleviate the degeneracy that exists between the mass, semimajor axis and eccentricity of the perturber, as we would now have a third dynamical constraint to consider in addition with the debris disk forced eccentricity and inner edge location. Indeed, assuming that circumplanetary material fills the perturber's Hill radius, the measurement of this source's extent would allow us to retrieve this quantity. From our observations, the source's extent is on average 0 ". 58 (13.8 au) in diameter, which would then correspond to 2 Hill radii of the perturber and we can thus write:

$$
2 a_{\mathrm{p}}\left(\frac{m_{\mathrm{p}}}{3 M_{\star}}\right)^{1 / 3}=13.8 \mathrm{au} .
$$

As this constraint depends on the mass and semimajor axis of the perturber, we display it in the right panel of Figure 14. One can then read the mass and semimajor axis of the perturber at the intersection and using in turn the constraint on the forced eccentricity, we can retrieve the corresponding perturber's eccentricity. In that case, the perturber would be characterized by $m_{\mathrm{p}}=0.8 M_{\mathrm{Jup}}, a_{\mathrm{p}}=107.5 \mathrm{au}$, and $e_{\mathrm{p}}=0.1$. Note that these constraints rely upon strong assumptions regarding the extent of the circumplanetary material, and that it could be expected that it does not entirely fill the Hill radius of the planet (Quillen \& Trilling 1998; Ayliffe \& Bate 2009), and consequently, the mass derived here for the perturber is only a lower mass limit. In addition, we did not take into account the error bars on the extent of the source, nor the fact that the source might be more extended than found by our best fit, as possibly suggested by the $2 \sigma$ residuals remaining around the source's location (see Figure 3). Our goal here is simply to show how the knowledge of a third constraint alleviates degeneracies between the mass and orbital properties of the perturber which are usually encountered when trying to characterize a perturber from its gravitational imprint on a debris ring. If this source's extent were larger than the $13.8 \mathrm{au}$ value we plugged in Equation (6), this would suggest that the planet bearing circumplanetary material is more massive than the $0.8 M_{\text {Jup }}$ value found here, which shall hence be considered a lower limit.

The origin of the only giant circumplanetary ring system we know of relatively well, namely that of Saturn, is still subject to debate, and in particular why Saturn harbors one and not the other giant planets. Three scenarios have been advanced to explain Saturn's ring system: (i) the tidal mass removal from a satellite migrating inwards toward Saturn (Canup 2010), (ii) the tidal disruption of cometary bodies during close encounters with Saturn (Dones 1991; Hyodo et al. 2017), and (iii) the collisional destruction of a satellite by a passing comet (Charnoz et al. 2009; Dubinski 2019). Scenario (i) is often preferred to scenarios (ii) and (iii), as these both rely on a significant cometary flux, and are therefore deemed fairly improbable in the case of Saturn. However, they certainly would be more plausible in the case the circumplanetary ring system has formed around a belt-shaping eccentric planet such as the one inferred around HD 202628. Indeed, when shaping the inner edge of a debris belt and clearing its chaotic zone, this type of perturber is extremely efficient at setting bodies onto cometary orbits that cross its own orbit (see Figure 6 of Faramaz et al. 2015), thus enhancing the probabilities of close approaches. While the majority of material is scattered rapidly, this dynamical phenomenon nevertheless reaches a steady state potentially ongoing over the system's lifetime, especially if one takes into account the fact that material will steadily diffuse into the planet's chaotic zone and re-feed it. Therefore, since HD 202628 is much older than either Fomalhaut and HR 4796, this phenomenon has most likely been ongoing for a much longer time, and there should be a greater probability for the scenarios (ii) and (iii) to have occurred around HD 202628, giving a potential explanation as to why this would be observed in the HD 202628 system, and not in the Fomalhaut and HR 4796 systems.

Another possible scenario could involve the trapping of dust that migrates inwards due to PR drag (Kennedy \& Piette 2015). However, small grains are more sensitive to PR drag, which would probably lead the perturber to trap small grains as well, which are not observed. Nevertheless, this scenario could still be compatible with the absence of small grains around the perturber, since these small grains cross more quickly and therefore could be trapped less efficiently. They could also tend to be collisionally destroyed more quickly, or might tend to stick together and grow again once bound to the planet.

\section{Summary and Conclusions}

In this work, we have presented a global, multi-wavelength view of the debris ring of HD 202628.

We performed high angular resolution $(\sim 0$ ". 8$)$ observations of the debris disk continuum with ALMA at $1.3 \mathrm{~mm}$, and searched for $\mathrm{CO}$ gas emission as well. In these observations, the star was detected with sufficient $\mathrm{S} / \mathrm{N}$ to provide accurate knowledge of its position, which is crucial to determine the ring's eccentricity. The ring appears narrower than seen with $H S T$, with a width of $\sim 1^{\prime \prime}$, that is, barely resolved with our $\sim 0$ ". 8 beam. We used a MCMC fit of our observations in the visibility space to determine the ring's geometry. The inner edge, inclination and position angle are compatible with those found in HST observations. Combining this with the knowledge of the star's position allowed us to confirm that the ring is intrinsically eccentric, with eccentricity $\sim 0.1$. This is somewhat smaller than what was originally found with HST by Krist et al. (2012).

No gas was detected. This allowed us to place upper limits on the gas content, as well as on the $\mathrm{CO}$ mass fraction of parent bodies. This last value is consistent with what was found in other debris belts. This means that $\mathrm{CO}$ could still be present at typical abundances without being detected, but the non detection tells us that any $\mathrm{CO}$ emission that may be present below the limits is optically thin and that gas has no influence on the debris ring dynamics.

Although this result remains qualitative, we find as well that the apocenter of the ring appears brighter than its pericenter, which is in accordance with what is expected of eccentric debris disks at these wavelengths and is called "apocenterglow". This brightness asymmetry due to an overdensity competes with another brightness asymmetry, the pericenterglow, which is a thermal effect due to the fact that the pericenter is closer to the star and thus hotter. At ALMA wavelengths, it is expected that the apocenter-glow dominates, while the pericenter-glow is instead expected to dominate at Herschel wavelengths. This is exactly what is seen in the 
Herschel observations at $70 \mu \mathrm{m}$, with the west side of the disk being brighter than the east side. This confirms the ring eccentric nature and the position of the ring pericenter on the west side of the ring. Finally, another feature that eccentric rings have been predicted to show in scattered light, as a result from the significant influence of radiation pressure on the micron-sized dust grains these observations trace, is a skirt at apastron. Our HST observations confirm this, as the ring extends further out on the apastron side than on the periastron side.

The photometric measurements provided by Herschel at both PACS and SPIRE wavelengths have allowed us, in combination with the ALMA ring photometry at $1.3 \mathrm{~mm}$, to derive the debris disk spectral index and constrain the grain-size distribution power-law index. This has been found to be 3.4, which allowed us to discard the possibility that planet formation was currently occurring in this debris disk, as this would require a power-law index of 4.5-5.5. Further SED modeling allowed us to determine the smallest grain size, $3.2 \mu \mathrm{m}$, which is larger than the blowout size, and to determine a fractional IR luminosity of $7 \times 10^{-5}$.

Focusing on the most simple scenario where a single planet interior to the ring shapes its edge and forces its eccentricity, we provide new constraints on the mass, semimajor axis and eccentricity of this putative perturber. Without an additional dynamical constraint, these constraints remain degenerate, as a small mass planet close to the ring inner edge would generate the same ring as a more massive and more eccentric perturber orbiting farther in from the ring toward the star. A third constraint that could alleviate this degeneracy would be some knowledge on the extent of the Hill radius of the perturber, and which could be retrieved if circumplanetary material was detected around the perturber. Our ALMA observations show several bright sources, and we considered the possibility that the source $S 1$ could be such material. Under this assumption, we show how precise constraints on the mass, semimajor axis and eccentricity of the perturber would be retrieved. Nevertheless, it cannot be excluded that this source is in fact a background object, which will be ascertained in the future with second epoch observations (Project 2018.1.00455.S, PI: V. Faramaz) to check for co-movement with the star. In addition, these new observations will be combined with those presented here, possibly leading to a more conclusive detection of the apocenter glow phenomenon.

We are very grateful for useful discussions with Mickael Bonnefoy and Julien Milli on exoplanets direct imaging, and with Eve Lee on the effects of radiation pressure on features seen in scattered light in debris disks. We are also very grateful to the anonymous referee for the constructive input. This paper makes use of the following ALMA data: ADS/JAO. ALMA\#2016.1.00515.S. ALMA is a partnership of ESO (representing its member states), NSF (USA) and NINS (Japan), together with NRC (Canada), MOST and ASIAA (Taiwan), and KASI (Republic of Korea), in cooperation with the Republic of Chile. The Joint ALMA Observatory is operated by ESO, AUI/NRAO and NAOJ. The National Radio Astronomy Observatory is a facility of the National Science Foundation operated under cooperative agreement by Associated Universities, Inc. V.F.'s postdoctoral fellowship is supported by the Exoplanet Science Initiative at the Jet Propulsion Laboratory, California Inst. of Technology, under
Table 7

Stellar Parameters for HD 202628

\begin{tabular}{|c|c|c|}
\hline Parameter & Value & Reference \\
\hline $\begin{array}{l}\alpha(\text { ICRS, epoch } \\
\text { 2015.5) }\end{array}$ & $319.61505582366 \mathrm{deg}$ & $\begin{array}{l}\text { Gaia Collaboration et al. } \\
\text { (2018) }\end{array}$ \\
\hline $\begin{array}{l}\delta \text { (ICRS, epoch } \\
2015.5 \text { ) }\end{array}$ & $-43.33455856542 \mathrm{deg}$ & $\begin{array}{l}\text { Gaia Collaboration et al. } \\
\text { (2018) }\end{array}$ \\
\hline Parallax & $41.9622 \pm 0.0455 \mathrm{mas}$ & $\begin{array}{l}\text { Gaia Collaboration et al. } \\
\text { (2018) }\end{array}$ \\
\hline Distance & $23.815 \pm 0.026 \mathrm{pc}$ & Bailer-Jones et al. (2018) \\
\hline Proper motion RA & $242.190 \pm 0.068$ mas yr $^{-1}$ & $\begin{array}{l}\text { Gaia Collaboration et al. } \\
\text { (2018) }\end{array}$ \\
\hline $\begin{array}{l}\text { Proper } \\
\text { motion Dec }\end{array}$ & $21.633 \pm 0.060 \mathrm{mas} \mathrm{yr}^{-1}$ & $\begin{array}{l}\text { Gaia Collaboration et al. } \\
\text { (2018) }\end{array}$ \\
\hline Radial velocity & $12.071 \pm 0.0027 \mathrm{~km} \mathrm{~s}^{-1}$ & $\begin{array}{l}\text { Gaia Collaboration et al. } \\
\text { (2018) }\end{array}$ \\
\hline$V$ & $6.742 \pm 0.004 \mathrm{mag}$ & $\begin{array}{l}\text { Mermilliod \& Nit- } \\
\text { schelm (1990) }\end{array}$ \\
\hline$G$ & $6.5825 \pm 0.0003 \mathrm{mag}$ & $\begin{array}{l}\text { Gaia Collaboration et al. } \\
\text { (2018) }\end{array}$ \\
\hline$M_{V}$ & $4.856 \pm 0.005 \mathrm{mag}$ & This study ${ }^{\mathrm{a}}$ \\
\hline Spec. Type & $\mathrm{G} 1.5 \mathrm{~V}$ & Gray et al. (2006) \\
\hline$T_{\text {eff }}$ & $5843 \pm 6 \mathrm{~K}$ & Spina et al. (2018) \\
\hline$[\mathrm{Fe} / \mathrm{H}]$ & $0.003 \pm 0.004$ & Spina et al. (2018) \\
\hline $\log (g)$ & $4.510 \pm 0.011$ & Spina et al. (2018) \\
\hline $\mathrm{U}$ & $-10.55 \pm 0.02 \mathrm{~km} \mathrm{~s}^{-1}$ & This study ${ }^{\mathrm{b}}$ \\
\hline V & $1.58 \pm 0.02 \mathrm{~km} \mathrm{~s}^{-1}$ & This study ${ }^{\mathrm{b}}$ \\
\hline W & $-28.05 \pm 0.02 \mathrm{~km} \mathrm{~s}^{-1}$ & This study ${ }^{\mathrm{b}}$ \\
\hline Mass(tracks) & $1.050 \pm 0.006 M_{\odot}$ & Spina et al. (2018) \\
\hline Mass(spectro.) & $1.068 \pm 0.038 M_{\odot}$ & This study ${ }^{c}$ \\
\hline $\log (\mathrm{L})$ & $-0.022 \pm 0.012 \mathrm{dex}$ & This study $^{\mathrm{c}}$ \\
\hline Radius & $0.951 \pm 0.013 R_{\odot}$ & This study $^{\mathrm{c}}$ \\
\hline
\end{tabular}

Notes.

${ }^{a}$ Calculated using Mermilliod \& Nitschelm (1990) V magnitude and Gaia DR2 parallax, assuming zero extinction.

${ }^{\mathrm{b}}$ Calculated using Gaia Collaboration et al. (2018) astrometry and mean published radial velocity.

${ }^{\mathrm{c}}$ Spectroscopic mass estimate adopts the surface gravity from Spina et al. (2018).

a contract with the National Aeronautics and Space Administration. M.B. acknowledges support from the Deutsche Forschungsgemeinschaft (DFG) through project Kr 2164/151. A.B., J.C., and J.O. acknowledge financial support from the ICM (Iniciativa Científica Milenio) via the Núcleo Milenio de Formación Planetaria grant. J.O. acknowledges financial support from the Universidad de Valparaíso, and from Fondecyt (grant 1180395). This work has made use of data from the European Space Agency (ESA) mission Gaia (https://www.cosmos.esa.int/gaia), processed by the Gaia Data Processing and Analysis Consortium (DPAC, https:// www.cosmos.esa.int/web/gaia/dpac/consortium). Funding for the DPAC has been provided by national institutions, in particular the institutions participating in the Gaia Multilateral Agreement.

\section{Appendix A \\ Age}

HD 202628 (HIP 105184) is a very well-characterized nearby young solar twin. Its stellar parameters are summarized in Table 7, and its published age Table 8 . The star is only slightly hotter than the Sun $\left(\mathrm{G} 1.5 \mathrm{~V}, T_{\text {eff }}=5833 \pm 6 \mathrm{~K}\right.$ Gray et al. 2006; Spina et al. 2018), with metallicity statistically 
Table 8

Age Estimates for HD 202628

\begin{tabular}{|c|c|c|}
\hline References & Age (Gyr) & Method \\
\hline Spina et al. (2018) & $0.6(0.3-1.1 ; 68 \% \mathrm{CL})$ & Isochronal \\
\hline Soto \& Jenkins (2018) & $2.809_{-1.505}^{+1.472}$ & Isochronal \\
\hline dos Santos et al. (2016) & $0.604 \pm 0.445$ & Isochronal \\
\hline $\begin{array}{l}\text { Reddy \& Lam- } \\
\text { bert (2017) }\end{array}$ & $0.4 \pm 0.4$ & Isochronal \\
\hline Krist et al. (2012) & $2.3 \pm 1$ & Chromospheric, X-ray \\
\hline $\begin{array}{l}\text { Casagrande et al. } \\
\qquad(2011)\end{array}$ & $\begin{array}{c}3.11(0.87-6.59 \\
68 \% \mathrm{CL})\end{array}$ & Isochronal(Padova) \\
\hline $\begin{array}{l}\text { Casagrande et al. } \\
\qquad(2011)\end{array}$ & $\begin{array}{c}3.41(0.96-7.06 \\
68 \% \mathrm{CL})\end{array}$ & Isochronal(BASTI) \\
\hline Holmberg et al. (2009) & $5.9(1.6-10.2 ; 68 \% \mathrm{CL})$ & Isochronal \\
\hline Takeda et al. (2007) & $0.64(0-3.8 ; 68 \% \mathrm{CL})$ & Isochronal \\
\hline $\begin{array}{l}\text { Valenti \& } \\
\text { Fischer (2005) }\end{array}$ & $5.0(1.8-6.8)$ & Isochronal \\
\hline $\begin{array}{l}\text { Rocha-Pinto et al. } \\
\text { (2004) }\end{array}$ & 2.27 & Chromospheric \\
\hline This study & 0.8 & {$[\mathrm{Y} / \mathrm{Mg}]^{\mathrm{a}}$} \\
\hline This study & 1.6 & Chromospheric $^{\mathrm{b}}$ \\
\hline This study & 1.0 & X-ray ${ }^{\mathrm{c}}$ \\
\hline adopted & $1.1 \pm 0.4$ & $\cdots$ \\
\hline
\end{tabular}

Notes.

${ }^{\text {a }}$ Using $[\mathrm{Y} / \mathrm{Mg}]$ abundance and age calibration from Tucci Maia et al. (2016).

${ }^{\mathrm{b}}$ Using median $\log \left(R_{H K}^{\prime}\right)=-4.67$ and calibration from Mamajek \& Hillenbrand (2008).

${ }^{\mathrm{c}}$ Using 2RXS X-ray flux from Boller et al. (2016) and calibration from Mamajek \& Hillenbrand (2008).

identical to solar $([\mathrm{Fe} / \mathrm{H}]=0.003 \pm 0.004)$ and surface gravity just slightly higher than solar $((\log (g)=4.51 \pm 0.01$ compared to 4.44 for Sun; Spina et al. 2018). This combination of slightly higher temperature and surface gravity led Spina et al. (2018) to estimate a young isochronal age $(0.6 \mathrm{Gyr} ; 0.3-1.1 \mathrm{Gyr}, 68 \%$ $\mathrm{CL})$ and slightly higher mass $\left(1.050 \pm 0.006 M_{\odot}\right)$. Other recent isochronal estimates give similarly young ages: $0.4 \pm 0.4 \mathrm{Gyr}$ (Reddy \& Lambert 2017), $0.604 \pm 0.445$ Gyr (Tucci Maia et al. 2016). However, as can be seen in Table 8, there are some recent estimates which place the age closer to $\sim 3 \mathrm{Gyr}$ (Casagrande et al. 2011; Soto \& Jenkins 2018), but those estimates have larger uncertainties.

There are multiple other age indicators which are similarly consistent with a young age for HD 202628. Besides the isochronal age, we discuss multiple age indicators for the star: $\mathrm{X}$-ray emission, chromospheric activity, and abundances of $\mathrm{Li}$, $\mathrm{Ba}$, and $\mathrm{Y}$. All of these are indicators are consistent with the star being slightly older than the Hyades cluster (recent estimates are consistent with $\sim 0.7 \pm 0.1$ Gyr; Brandt \& Huang 2015; Gossage et al. 2018; Martín et al. 2018; Salaris $\&$ Bedin 2018) and significantly younger than the Sun (4.567 Gyr; Amelin et al. 2010). Krist et al. (2012) reviewed the choromospheric and X-ray activity indicators and adopted an age of $2.3 \pm 1$ Gyr.

As Sun-like stars deplete their Li as they age, trends among age-dated solar twins show that HD 202628's high Li abundance $(\log \epsilon(\mathrm{Li}) \mathrm{LTE}=2.23 \pm 0.02)$ would similarly appear unusual if the star were $>2$ Gyr (see Figure 4 of Reddy \& Lambert 2017), but appears to be clearly older than the $\sim 0.7$ Gyr old Hyades (see Figure 6 of Sestito et al. 2003). Recent spectroscopic surveys of solar twins have shown evidence of strong age-dependent trends in $s$-process elements (e.g., Spina et al. 2016, 2018; Tucci Maia et al. 2016; Reddy \& Lambert 2017). The star's high barium abundance ([Ba/ $\mathrm{Fe}]=+0.24)$ is typical for young solar twins, and would appear anomalously high if the star were $>2$ Gyr (see Figure 5 of Reddy \& Lambert 2017). Through comparison to an abundance-ratio versus age trend for age-dated solar twins, the [Y/Mg] abundance ratio for HD 202628 (0.152 \pm 0.015$)$ derived by Tucci Maia et al. (2016) is consistent with an age of 0.8 Gyr.

The star's chromospheric activity indicator $\left(\log R_{H K}^{\prime}\right)$ has been reported by multiple surveys, with a wide range of values (maximum of -4.61 reported by Jenkins et al. 2006 to minimum of -4.782 reported by Gray et al. 2006). Drawing upon the published activity values from Henry et al. (1996), Tinney et al. (2002), Jenkins et al. (2006), Gray et al. (2006), Cincunegui et al. (2007), Murgas et al. (2013), Reddy \& Lambert (2017), Meunier et al. (2017), and Saikia et al. (2018), we adopt a median estimate of $\log R_{H K}^{\prime}=-4.67$. Using the chromospheric activity-age calibration of Mamajek \& Hillenbrand (2008), this is consistent with an age of $1.7 \mathrm{Gyr}$, whereas converting the activity to a predicted rotation period (14 days) would predict an age of 1.6 Gyr. The star's soft X-ray emission was detected in the ROSAT All-Sky Survey (Voges et al. 1999). Based on the recent reduction of the ROSAT data by (Boller et al. 2016; 2RXS catalog), we estimate an X-ray activity level of $\log \left(L_{\mathrm{X}} / L_{\mathrm{bol}}\right)=-5.14$ and X-ray luminosity $L_{\mathrm{X}}=10^{28.42}$ $\mathrm{erg} \mathrm{s}^{-1}$. Using the X-ray versus age calibration of Mamajek \& Hillenbrand (2008), this corresponds to an age of $1.2 \mathrm{Gyr}$, whereas converting the $\mathrm{X}$-ray flux to a predicted rotation period (10.2 days) and then to age via gyrochronology, results in an age of $1.0 \mathrm{Gyr}$. Taking into account the constraints on the age based on the previously discussed abundance data, the isochronal estimate from Spina et al. (2018), and the coronal and chromospheric activity age estimates, we adopt a final age of $1.1 \pm 0.4 \mathrm{Gyr}$.

To improve estimates of the star's luminosity and radius, and verify the star's mass, we take advantage of the star's similarity to the Sun to derive improved parameters. HD 202628 is only $61 \mathrm{~K}$ hotter than the Sun. While different Bolometric Correction (BC) compilations sometimes have sizeable zero-point differences (Torres 2010), the scales (e.g., Flower 1996; Bessell et al. 1998; Casagrande et al. 2008) are in excellent agreement that the star's $\mathrm{BC}_{V}$ value should be $\Delta \mathrm{BC}_{V}=\mathrm{BC}_{V}-$ $\mathrm{BC}_{V, \odot} \simeq 0.01 \mathrm{mag}$ (few mmag uncertainty) relative to that for the solar effective temperature $\left(\mathrm{T}_{\text {eff }, \odot}=5772 \mathrm{~K}\right.$; IAU 2015 nominal value). We write the bolometric magnitude equation relative to the Sun:

$$
M_{\mathrm{bol}}=M_{\mathrm{bol}, \odot}+M_{V}-M_{V, \odot}+\Delta B C_{V} .
$$

Adopting the IAU 2015 nominal solar values and bolometric magnitude (IAU 2015 bolometric magnitude scale is calibrated to nominal solar $M_{\text {bol, } \odot}=4.74$, tied to nominal solar luminosity and $3.828 \pm 10^{26} \mathrm{~W}$ and nominal total solar irradiance $S_{\odot}=1361 \mathrm{~W} \mathrm{~m}^{-2}$; Mamajek et al. 2015), ${ }^{18}$ the solar apparent $V$ magnitude from (Torres 2010, $\left.V_{\odot}=-26.76 \pm 0.03\right)$ which corresponds to solar $M_{V, \odot}=4.812 \pm 0.03$, we estimate $M_{\mathrm{bol}}=4.794 \pm 0.03$. Remarkably, the uncertainty is completely dominated by that of the solar $V$ magnitude. This translates to luminosity $\log \left(L / L_{\odot}\right)=-0.022 \pm 0.012$, i.e., $95 \% \pm 3 \%$ that of the

\footnotetext{
18 https://www.iau.org/static/resolutions/IAU2015_English.pdf
} 


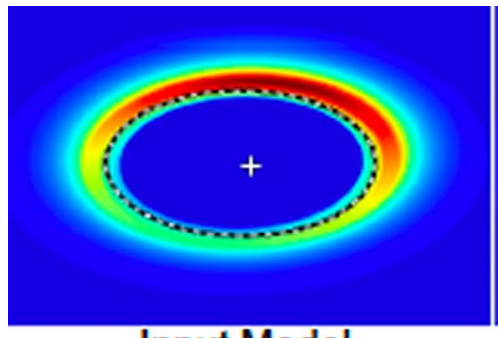

Input Model

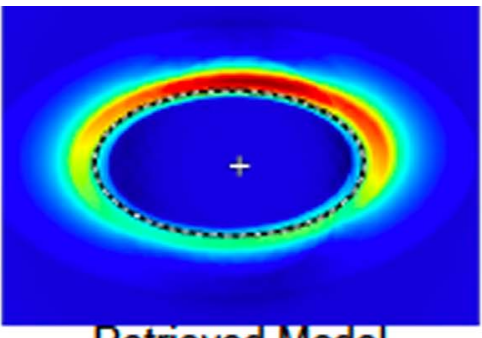

Retrieved Model

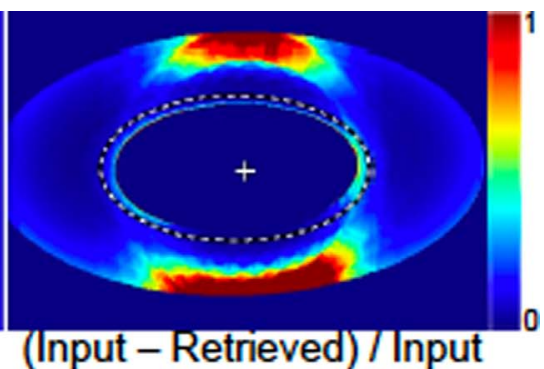

(Input - Retrieved)//nput

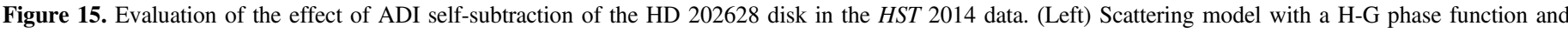

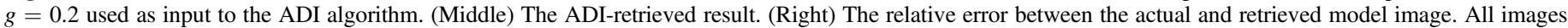
are shown from minimum to maximum value on a linear scale.

Sun. Given the well-defined effective temperature from Spina et al. (2018), this luminosity corresponds to a photospheric radius of $0.951 \pm 0.013 R_{\odot}$. One can derive a spectroscopic estimate of the star's mass based on the precise estimates of surface gravity and radius. Adopting $\log (g)=4.438$ for the Sun, we estimate the mass to be $\log \left(M / M_{\odot}\right)=0.028 \pm 0.015$ or $1.068 \pm 0.038 M_{\odot}$. This corresponds well to the recent estimate based on evolutionary tracks by Spina et al. (2018) of $1.050 \pm 0.006 M_{\odot}$, and empirically confirms the star to be slightly more massive than the Sun independent of theoretical evolutionary models.

\section{Appendix B \\ Impact of Disk Self-subtraction due to ADI Processing in HST Observations}

The ADI post-processing method used here to remove the stellar instrumental pattern from the HST images has a major drawback with extended sources like circumstellar disks: the possibility of source self-subtraction. The algorithm assumes that the stellar PSF pattern on the detector is constant regardless of the telescope orientation. Any variation in a given pixel between images is then due to an astronomical source moving through that pixel as the telescope is rotated about the star. The mean signal that rotates with the same orientation as the telescope then constitutes the derived source image.

For small, isolated sources like an exoplanet or small galaxy and with sufficient roll, no pixel sees the same astronomical source more than once, and its signal is fully assigned to the source image. But if the object is sufficiently extended, like a disk, such that parts of it pass through a given pixel at two or more orientations, then a portion of that signal will be considered constant and part of the PSF. These over-bright regions of the PSF would then cause oversubtraction of the PSF, or effectively self-subtraction of the source. This can be minimized by maximizing the differences between, and number of, orientations. In the case of a uniform, face-on disk, however, the disk will fully self-subtract itself.

To evaluate the potential for self-subtraction, the $g=0.2$ scattered light model was added to copies of the same uniformintensity image (the PSF) at the same nine orientations used in the 2014 observations and sent through the ADI algorithm. Since this PSF is constant with roll, this experiment solely evaluates the impact of self-subtraction. The results are shown in Figure 15 and demonstrate that self-subtraction is minimal $(<15 \%)$ over the majority of the visible disk, including those regions along the line of sight and closest to the star. Significant $(>80 \%)$ errors occur in the outer, farthest reaches of the disk along the line of sight.

\section{ORCID iDs}

Karl R. Stapelfeldt (1D https://orcid.org/0000-0002-2805-7338

Eric E. Mamajek (i) https://orcid.org/0000-0003-2008-1488

Luca Matrà (iD https://orcid.org/0000-0003-4705-3188

Mark Booth (10) https://orcid.org/0000-0001-8568-6336

Kevin Flaherty (i) https://orcid.org/0000-0003-2657-1314

Antonio S. Hales (i) https://orcid.org/0000-0001-5073-2849

Amelia Bayo (iD https://orcid.org/0000-0001-7868-7031

Simon Casassus (i) https://orcid.org/0000-0002-0433-9840

Johan Olofsson (ib https://orcid.org/0000-0003-4475-3605

Kate Y. L. Su (ib https://orcid.org/0000-0002-3532-5580

David J. Wilner (i) https://orcid.org/0000-0003-1526-7587

\section{References}

Acke, B., Min, M., Dominik, C., et al. 2012, A\&A, 540, A125

Amelin, Y., Kaltenbach, A., Iizuka, T., et al. 2010, E\&PSL, 300, 343

Andrews, S. M., Rosenfeld, K. A., Kraus, A. L., \& Wilner, D. J. 2013, ApJ, 771,129

Andrews, S. M., \& Williams, J. P. 2005, ApJ, 631, 1134

Aumann, H. H., Gillett, F. C., Beichman, C. A., et al. 1984, ApJL, 278, L23

Ayliffe, B. A., \& Bate, M. R. 2009, MNRAS, 397, 657

Bailer-Jones, C. A. L., Rybizki, J., Fouesneau, M., Mantelet, G., \& Andrae, R. 2018, AJ, 156, 58

Baraffe, I., Chabrier, G., Barman, T. S., Allard, F., \& Hauschildt, P. H. 2003 , A\&A, 402, 701

Becker, T. M., Colwell, J. E., Esposito, L. W., \& Bratcher, A. D. 2016, Icar, 279, 20

Beckwith, S. V. W., Sargent, A. I., Chini, R. S., \& Guesten, R. 1990, AJ, 99, 924

Bessell, M. S., Castelli, F., \& Plez, B. 1998, A\&A, 333, 231

Bocchio, M., Bianchi, S., \& Abergel, A. 2016, A\&A, 591, A117

Boley, A. C., Payne, M. J., Corder, S., et al. 2012, ApJL, 750, L21

Boller, T., Freyberg, M. J., Trümper, J., et al. 2016, A\&A, 588, A103

Bonnefoy, M., Perraut, K., Lagrange, A.-M., et al. 2018, A\&A, 618, A63

Booth, M., Kennedy, G., Sibthorpe, B., et al. 2013, MNRAS, 428, 1263

Brandt, T. D., \& Huang, C. X. 2015, ApJ, 807, 58

Canup, R. M. 2010, Natur, 468, 943

Carniani, S., Maiolino, R., De Zotti, G., et al. 2015, A\&A, 584, A78

Casagrande, L., Flynn, C., \& Bessell, M. 2008, MNRAS, 389, 585

Casagrande, L., Schönrich, R., Asplund, M., et al. 2011, A\&A, 530, A138

Charnoz, S., Morbidelli, A., Dones, L., \& Salmon, J. 2009, Icar, 199, 413

Chiang, E., Kite, E., Kalas, P., Graham, J. R., \& Clampin, M. 2009, ApJ, 693,734

Cincunegui, C., Díaz, R. F., \& Mauas, P. J. D. 2007, A\&A, 469, 309

Cutri, R. M., Skrutskie, M. F., van Dyk, S., et al. 2003, 2MASS All Sky Catalog of Point Sources (Washington, DC: NASA)

Cutri, R. M., Wright, E. L., Conrow, T., et al. 2012, yCat, 2311, 0

Daley, C., Hughes, A. M., Carter, E. S., et al. 2019, ApJ, 875, 87

Dohnanyi, J. S. 1969, JGR, 74, 2531

Dones, L. 1991, Icar, 92, 194

dos Santos, L. A., Meléndez, J., do Nascimento, J.-D., et al. 2016, A\&A, 592, A156

Draine, B. T. 1978, ApJS, 36, 595

Dubinski, J. 2019, Icar, 321, 291 
Faramaz, V., Beust, H., Augereau, J.-C., Kalas, P., \& Graham, J. R. 2015, A\&A, 573, A87

Faramaz, V., Beust, H., Thébault, P., et al. 2014, A\&A, 563, A72

Flaherty, K. M., Hughes, A. M., Rosenfeld, K. A., et al. 2015, ApJ, 813, 99

Flower, P. J. 1996, ApJ, 469, 355

Foreman-Mackey, D., Hogg, D. W., Lang, D., \& Goodman, J. 2013, PASP, 125,306

Gaia Collaboration, Brown, A. G. A., Vallenari, A., et al. 2018, A\&A, 616, A1

Gaia Collaboration, Prusti, T., de Bruijne, J. H. J., et al. 2016, A\&A, 595, A1

Golimowski, D. A., Krist, J. E., Stapelfeldt, K. R., et al. 2011, AJ, 142, 30

Gossage, S., Conroy, C., Dotter, A., et al. 2018, ApJ, 863, 67

Gray, R. O., Corbally, C. J., Garrison, R. F., et al. 2006, AJ, 132, 161

Hedman, M. M., \& Stark, C. C. 2015, ApJ, 811, 67

Henry, T. J., Soderblom, D. R., Donahue, R. A., \& Baliunas, S. L. 1996, AJ, 111,439

Henyey, L. G., \& Greenstein, J. L. 1941, ApJ, 93, 70

Holmberg, J., Nordström, B., \& Andersen, J. 2009, A\&A, 501, 941

Hong, S. S. 1985, A\&A, 146, 67

Hughes, A. M., Duchêne, G., \& Matthews, B. C. 2018, ARA\&A, 56, 541

Hyodo, R., Charnoz, S., Ohtsuki, K., \& Genda, H. 2017, Icar, 282, 195

Jackson, A. P., Wyatt, M. C., Bonsor, A., \& Veras, D. 2014, MNRAS, 440,3757

Jenkins, J. S., Jones, H. R. A., Tinney, C. G., et al. 2006, MNRAS, 372, 163

Kalas, P., Graham, J. R., \& Clampin, M. 2005, Natur, 435, 1067

Kennedy, G. M., \& Piette, A. 2015, MNRAS, 449, 2304

Kenworthy, M. A., \& Mamajek, E. E. 2015, ApJ, 800, 126

Kenyon, S. J., \& Bromley, B. C. 2015, ApJ, 806, 42

Koerner, D. W., Kim, S., Trilling, D. E., et al. 2010, ApJL, 710, L26

Kral, Q., Thébault, P., \& Charnoz, S. 2013, A\&A, 558, A121

Krist, J. E. 2004, Proc. SPIE, 5487, 1284

Krist, J. E., Stapelfeldt, K. R., Bryden, G., et al. 2010, AJ, 140, 1051

Krist, J. E., Stapelfeldt, K. R., Bryden, G., \& Plavchan, P. 2012, AJ, 144, 45

Krivov, A. V. 2010, RAA, 10, 383

Lee, E. J., \& Chiang, E. 2016, ApJ, 827, 125

Liseau, R., De la Luz, V., O’Gorman, E., et al. 2016, A\&A, 594, A109

Lyra, W., \& Kuchner, M. 2013, Natur, 499, 184

MacGregor, M. A., Wilner, D. J., Chandler, C., et al. 2016, ApJ, 823, 79

Mamajek, E. E. 2012, ApJL, 754, L20

Mamajek, E. E., \& Hillenbrand, L. A. 2008, ApJ, 687, 1264

Mamajek, E. E., Prsa, A., Torres, G., et al. 2015, arXiv:1510.07674

Mamajek, E. E., Quillen, A. C., Pecaut, M. J., et al. 2012, AJ, 143, 72

Marino, S., Wyatt, M. C., Panić, O., et al. 2017, MNRAS, 465, 2595

Martín, E. L., Lodieu, N., Pavlenko, Y., \& Béjar, V. J. S. 2018, ApJ, 856, 40

Matrà, L., Dent, W. R. F., Wyatt, M. C., et al. 2017a, MNRAS, 464, 1415

Matrà, L., MacGregor, M. A., Kalas, P., et al. 2017b, ApJ, 842, 9

Matrà, L., Panić, O., Wyatt, M. C., \& Dent, W. R. F. 2015, MNRAS, 447, 3936

Matrà, L., Wilner, D. J., Öberg, K. I., et al. 2018, ApJ, 853, 147
McMullin, J. P., Waters, B., Schiebel, D., Young, W., \& Golap, K. 2007, in ASP Conf. Ser. 376, Astronomical Data Analysis Software and Systems XVI, ed. R. A. Shaw, F. Hill, \& D. J. Bell (San Francisco, CA: ASP), 127 Mermilliod, J. C., \& Nitschelm, C. 1990, A\&AS, 84, 133

Meunier, N., Mignon, L., \& Lagrange, A.-M. 2017, A\&A, 607, A124

Moerchen, M. M., Churcher, L. J., Telesco, C. M., et al. 2011, A\&A, 526, A34

Moro-Martin, A. 2013, in Planets, Stars and Stellar Systems, ed. T. D. Oswalt, L. M. French, \& P. Kalas (Dordrecht: Springer), 431

Murgas, F., Jenkins, J. S., Rojo, P., Jones, H. R. A., \& Pinfield, D. J. 2013, A\&A, 552, A27

Pan, M., Nesvold, E. R., \& Kuchner, M. J. 2016, ApJ, 832, 81

Pawellek, N., \& Krivov, A. V. 2015, MNRAS, 454, 3207

Pawellek, N., Krivov, A. V., Marshall, J. P., et al. 2014, ApJ, 792, 65

Pearce, T. D., \& Wyatt, M. C. 2014, MNRAS, 443, 2541

Quillen, A. C. 2006, MNRAS, 372, L14

Quillen, A. C., \& Trilling, D. E. 1998, ApJ, 508, 707

Reddy, A. B. S., \& Lambert, D. L. 2017, ApJ, 845, 151

Ricci, L., Testi, L., Maddison, S. T., \& Wilner, D. J. 2012, A\&A, 539, L6

Rocha-Pinto, H. J., Flynn, C., Scalo, J., et al. 2004, A\&A, 423, 517

Rodigas, T. J., Malhotra, R., \& Hinz, P. M. 2014, ApJ, 780, 65

Rosenfeld, K. A., Andrews, S. M., Hughes, A. M., Wilner, D. J., \& Qi, C. 2013, ApJ, 774, 16

Saikia, S. B., Marvin, C. J., Jeffers, S. V., et al. 2018, A\&A, 616, A108

Salaris, M., \& Bedin, L. R. 2018, MNRAS, 480, 3170

Schneider, G., Grady, C. A., Stark, C. C., et al. 2016, AJ, 152, 64

Sestito, P., Randich, S., Mermilliod, J.-C., \& Pallavicini, R. 2003, A\&A, 407, 289

Sierchio, J. M., Rieke, G. H., Su, K. Y. L., \& Gáspár, A. 2014, ApJ, 785, 33

Soto, M. G., \& Jenkins, J. S. 2018, A\&A, 615, A76

Spina, L., Meléndez, J., Karakas, A. I., et al. 2016, A\&A, 593, A125

Spina, L., Meléndez, J., Karakas, A. I., et al. 2018, MNRAS, 474, 2580

Stark, C. C., Schneider, G., Weinberger, A. J., et al. 2014, ApJ, 789, 58

Takeda, G., Ford, E. B., Sills, A., et al. 2007, ApJS, 168, 297

Tempel, E., Tago, E., \& Liivamägi, L. J. 2012, A\&A, 540, A106

Thébault, P., \& Augereau, J.-C. 2007, A\&A, 472, 169

Thebault, P., Kral, Q., \& Ertel, S. 2012, A\&A, 547, A92

Thilliez, E., \& Maddison, S. T. 2016, MNRAS, 457, 1690

Tinney, C. G., McCarthy, C., Jones, H. R. A., et al. 2002, MNRAS, 332, 759

Torres, G. 2010, AJ, 140, 1158

Tucci Maia, M., Ramírez, I., Meléndez, J., et al. 2016, A\&A, 590, A32

Valenti, J. A., \& Fischer, D. A. 2005, ApJS, 159, 141

van Dishoeck, E. F., Jonkheid, B., \& van Hemert, M. C. 2006, FaDi, 133, 231 van Leeuwen, F. 2007, A\&A, 474, 653

Visser, R., van Dishoeck, E. F., \& Black, J. H. 2009, A\&A, 503, 323

Voges, W., Aschenbach, B., Boller, T., et al. 1999, A\&A, 349, 389

Wyatt, M. C. 1999, PhD thesis, Royal Observatory, Blackford Hill

Wyatt, M. C. 2008, ARA\&A, 46, 339

Wyatt, M. C., Dermott, S. F., Telesco, C. M., et al. 1999, ApJ, 527, 918 